\title{
Immune Response and Pathogen Invasion at the Choroid Plexus in the Onset of Cerebral Toxoplasmosis
}

Caio Andreeta Figueiredo

OvGU Medizinische Fakultät

JOhannes Steffen

OvGU Medizinische Fakultät

Lorena Morton

OvGU Medizinische Fakultät

\section{Sushmita Arumugam}

OvGU Medizinische Fakultät

\section{OLiver Liesenfeld}

Charite University Hospital Berlin

\section{Maria A Deli}

Hungarian Academy of Science Biological Research Centre

\section{Andrea Kröger}

OvGU Medizinische Fakultät

Thomas Schüler

OvGU Medizinische Fakultät

Ildiko Rita Dunay ( $\square$ ildikodunay@gmail.com )

Otto von Guericke Universitat Magdeburg https://orcid.org/0000-0002-9900-8605

\section{Research Article}

Keywords: Toxoplasma gondii, neuroinflammation, choroid plexus, blood-CSF barrier, blood-brain barrier, tight junctions, metalloproteinases.

Posted Date: September 27th, 2021

DOI: https://doi.org/10.21203/rs.3.rs-910513/v1

License: (c) (1) This work is licensed under a Creative Commons Attribution 4.0 International License.

Read Full License 
Version of Record: A version of this preprint was published at Journal of Neuroinflammation on January 13th, 2022. See the published version at https://doi.org/10.1186/s12974-021-02370-1. 


\section{Immune response and pathogen invasion at the choroid plexus in the onset of cerebral toxoplasmosis}

3 Caio Andreeta Figueiredo', Johannes Steffen ${ }^{1}$, Lorena Morton ${ }^{1}$, Sushmita Arumugam ${ }^{1}$, 4 Oliver Liesenfeld ${ }^{2}$, Mária A Deli $^{3}$, Andrea Kröger ${ }^{4}$, Thomas Schüler ${ }^{5}$, Ildiko Rita 5 Dunay*1,6

$6{ }^{1}$ Institute of Inflammation and Neurodegeneration, Medical Faculty, Otto-von-Guericke

7 University Magdeburg, Magdeburg, Germany

$8 \quad{ }^{2}$ Institute for Microbiology and Hygiene, Charité Medical School, Berlin, Germany.

$9 \quad{ }^{3}$ Institute of Biophysics, Biological Research Centre, H-6726, Szeged, Hungary;

$10{ }^{4}$ Institut for Medical Microbiology and Hospital Hygiene, Medical Faculty, Otto-von-Guericke 11 University Magdeburg, Magdeburg, Germany

$12{ }^{5}$ Institute of Molecular and Clinical Immunology, Medical Faculty, Otto-von-Guericke 13 University Magdeburg, Magdeburg, Germany

$14{ }^{6}$ Center for Behavioral Brain Sciences - CBBS - Magdeburg, Germany

15 * Correspondence:

16 Ildiko Rita Dunay

17 ildikodunay@gmail.com

18 Abstract

Background: Toxoplasma gondii (T. gondii) is a highly successful parasite being able to cross all biological barriers of the body, finally reaching the central nervous system (CNS). Previous studies have highlighted the critical involvement of the blood-brain barrier (BBB) during $T$. gondii invasion and development of subsequent neuroinflammation. Still, the potential contribution of the choroid plexus (CP), a main structure forming the blood-cerebrospinal fluid (CSF)-barrier (BCSFB) have not been addressed.

Methods: To investigate $T$. gondii invasion and the onset of neuroinflammation, the CP and brain microvessels (BMV) were isolated and analysed for parasite burden. Additionally, immuno-stained brain sections and three dimensional whole mount preparations were evaluated for parasite localization and morphological alterations. Activation of choroidal and brain endothelial cells were characterized by flow cytometry. To evaluate the impact of early immune 
responses on $\mathrm{CP}$ and $\mathrm{BMV}$, expression levels of inflammatory mediators, tight junctions (TJ) and matrix metalloproteinases (MMPs) were quantified. Additionally, FITC-dextran was applied to determine infection-related changes in BCSFB permeability. Finally, the response of primary CP epithelial cells to $T$. gondii parasites was tested in vitro.

Results: Here we revealed that endothelial cells in the CP are initially infected by $T$. gondii, and become activated prior to BBB endothelial cells indicated by MHCII upregulation. Additionally, CP elicited early local immune response with upregulation of IFN- $\gamma$, TNF, IL-6, host-defence factors as well as swift expression of CXCL9 chemokine, when compared to the BMV. Consequently, we uncovered distinct TJ disturbances of claudins, associated with upregulation of MMP-8 and MMP-13 expression in infected CP in vivo, which was confirmed by in vitro infection of primary CP epithelial cells. Notably, we detected early barrier damage and functional loss by increased BCSFB permeability to FITC-dextran in vivo, which was extended over the infection course.

Conclusions: Altogether, our data reveal a close interaction between $T$. gondii infection at the $\mathrm{CP}$ and the impairment of the BCSFB function indicating that infection-related neuroinflammation is initiated in the $\mathrm{CP}$.

Keywords: Toxoplasma gondii, neuroinflammation, choroid plexus, blood-CSF barrier, bloodbrain barrier, tight junctions, metalloproteinases.

\section{Background}

Toxoplasmosis is a foodborne parasitic disease caused by the obligate intracellular protozoan Toxoplasma gondii (T. gondii). It is estimated that more than one-third of the world's human population is infected with $T$. gondii and its seroprevalence increases gradually with age (1-3). Following uptake via oral ingestion of contaminated food or water, the parasites proliferate within a variety of nucleated cells, infect circulating leukocytes, successfully cross all the barriers of the body and spread throughout host tissues (4). Despite of the central nervous 
system (CNS) being an immune privileged site shielded from peripheral infections and inflammation, $T$. gondii parasites are able to invade the CNS. Previous studies have proposed different mechanisms of parasite invasion, including: (i) active paracellular migration of free parasites, (ii) transmigration of hypermotile infected leukocytes, defined as "Trojan horse" mechanism, and (iii) infection and replication of parasites within brain endothelial cells (5-12). Once parasites cross the brain biological barriers, e.g. blood-brain-barrier (BBB), they invade brain-resident cells and persist in cysts lifelong (13-15). The cyst formation within neurons develops a stress-mediated response followed by ongoing basal neuroinflammation, which leads to altered neuronal function, and potentially to behavioral alterations and neuropsychiatric diseases (16-19). In immunodeficient individuals, the infection can lead to disruption of tissue cysts and uncontrolled parasite proliferation, resulting in toxoplasma encephalitis (TE) $(2,20$, 21).

Several studies have demonstrated the decisive involvement of the BBB in the invasion of $T$. gondii and the ensuing development of neuroinflammation (reviewed by $(22,23)$ ). In fact, the choroid plexus (CP) is another barrier and potential interface for pathogen invasion into CNS. The CP is the main structure forming the blood-CSF-barrier (BCSFB), and is crucial for CNS homeostasis and cerebrospinal fluid (CSF) secretion. Located within the four brain ventricles, the $\mathrm{CP}$ is a villous and selective organ formed by adjacent epithelial cells anchored to a basal lamina and an inner core of resident immune cells surrounding a dense vascular network of fenestrated endothelial cells. Choroidal epithelial cells are tightly interconnected by tight junction proteins (TJ), and control the molecular and cellular composition of the CSF $(24,25)$. The ability of this unique neuro-immune interface to actively integrate signals between brain and periphery is fundamental to CNS immunity (26), in which CP regulation of immune cell trafficking is considered a central point in the initiation of inflammatory brain responses (27). Alterations or even disruption of the CP epithelium in response to stressful events have detrimental effects on barrier permeability compromising the BCSFB functions (28). Indeed, 
81 BCSFB breakdown has been implicated in neurodegenerative diseases (29-33) and during infection-induced inflammation $(31,34)$, often indicating the involvement of matrix metalloproteinases (MMPs). Moreover, recent studies have described that the BCSFB serves as a hotspot for direct pathogen infiltration into the CNS (35). However, data concerning the contribution of the $\mathrm{CP}$ to $T$. gondii invasion, and subsequent neuroinflammation are controversial and incomplete. For example, the analysis of postmortem samples from immunodeficient patients with cerebral toxoplasmosis identified the $\mathrm{CP}$ as a site of infection (36). On the contrary, models of reactivated TE indicate no evidence for the involvement of CP in systemic parasitic dissemination $(37,38)$.

Here, we demonstrate that, prior to the activation of endothelial cells in the BBB, parasites invading the CNS rapidly seize choroidal endothelial cells. As a result, a prompt immune response is initiated in the $\mathrm{CP}$ as shown by the upregulation of cytokines, chemokines, and host-defense-factors, followed by the expression of MMPs, TJ disturbance of CP epithelial cells, and subsequent increased barrier permeability. Together, our results show that the infection takes place in the $\mathrm{CP}$, and indicate sudden functional impairment of the BCSFB upon the onset of the CNS invasion by $T$. gondii.

\section{Methods}

\subsection{Mice and infections in vivo}

Experiments were conducted with female C57BL/6J mice (8-14 weeks old, purchased from Janvier, Cedex, France). All animals were group-housed in a $12 \mathrm{~h}$ day/night cycle at $22^{\circ} \mathrm{C}$ with free access to food and water under specific-pathogen-free conditions and according to institutional guidelines approved by the Animal Studies Committee of Saxony-Anhalt. In order to investigate early $T$. gondii infection, mice were infected by intraperitoneal (i.p.) injection of either cysts or tachyzoites. For cyst infection, 2 cysts of the type II ME49 strain harvested from the brains of female NMRI mice infected i.p. with $T$. gondii cysts 6-12 months earlier were used 
106 as previously described (39). For tachyzoites infection, type II T. gondii reporter parasites of 107 the PTG-GFPS65T strain were grown in monolayers of human foreskin fibroblast (HFF) with

108 DMEM medium (FG0435, Biochrom, Germany), supplemented with 10\% fetal bovine serum 109 (FBS) (ThermoFisher, Germany), 1\% Penicillin/Streptomycin (Pen/Strep; Sigma, USA) and $1101 \%$ non-essential amino acids (NEEA) (ThermoFisher, Germany) as previously described (40).

111 Freshly egressed parasites were filtered through a $5 \mu \mathrm{m}$ Millex-SV syringe filter (Millipore, 112 Germany), and the number of living tachyzoites determined by counting under a light 113 microscope using Trypan Blue 0.4\%. Mice were infected i.p. with 1 x $10^{5}$ reporter parasites in $114200 \mu 1$ PBS.

\section{2.2. Organ isolation}

Mice were deeply anaesthetized by isofluorane inhalation (Baxter), the CSF was

117 collected and thereafter animals were transcardially perfused with $60 \mathrm{ml}$ PBS. For

118 immunofluorescence samples, perfusion was additionally done with $20 \mathrm{ml}$ of $4 \%$ 119 paraformaldehyde (PFA) in PBS. Brain, spleen and spinal cord were removed and stored in 120 sterile ice-cold PBS or RNAlater (Sigma) for further processing. Samples stored in RNAlater 121 were kept at $4{ }^{\circ} \mathrm{C}$ overnight and afterwards transferred to $-80{ }^{\circ} \mathrm{C}$.

\section{2.3. Cerebrospinal fluid collection}

CSF was collected by the cisterna magna puncture technique as described elsewhere

124 (41). In short, deeply anaesthetized animals were immobilized in a prone position with the head

125 forming a $135^{\circ}$ angle with the body, and a sagittal incision of the skin was made inferior to the occiput. Using a stereomicroscope (Stemi 305; ZEISS), the subcutaneous tissue and muscles were dissected, and a glass capillary tube was introduced into the cisterna magna through the 
129 collected, and kept on ice until further processing. Samples were macroscopically assessed for

130 blood contamination, and discarded when contamination was detected.

\subsection{Choroid plexus and brain tissue isolation}

Isolated brains were placed under a stereomicroscope in dissection buffer containing

133 HBSS (ROTI ${ }^{\circledR}$ Cell, Roth), and 10 mM HEPES (Gibco, ThermoFisher). CPs were isolated from

134 the lateral, third and fourth brain ventricles, and were either processed for total RNA/DNA

135 isolation, or placed in digestion buffer for further cell isolation. Cerebellum, olfactory bulbs,

136 and adjacent brain meninges were removed and discarded. The remaining brain tissue was used

137 for cell isolation followed by flow cytometric analysis, or further utilized for brain microvessels

138 isolation.

139

140

142

143

144

145

146

147 pellet containing microvessels was re-suspended in PBS, fractioned on $22 \%(\mathrm{v} / \mathrm{v})$ Percoll®

148 (Sigma, \#GE17089101) gradient solution and centrifuged for $10 \mathrm{~min}, 600 \mathrm{~g}$, w/o brake. The

\subsection{Brain microvessels isolation}

Brain microvessels were isolated as previously described (42) with a few modifications, and used for immunofluorescence, or total RNA/DNA isolation. Briefly, brain hemispheres were minced with a scalpel, and homogenized in digestion buffer (HBSS, with $6.75 \mathrm{~g} / 1$ glucose, $20 \mathrm{mM}$ HEPES) containing $1 \mathrm{mg} / \mathrm{ml}$ DNAse I. After incubation $\left(10 \mathrm{~min}, 37^{\circ} \mathrm{C}\right)$, homogenate was washed in FACS buffer (PBS w/o Ca/Mg, 2 mM EDTA, 2\% v/v FBS, 10 mM HEPES). The resultant pellet was re-suspended and separated by successive centrifugation in $20 \%(\mathrm{w} / \mathrm{v})$ bovine serum albumin (BSA)-DMEM/F12 solution. To remove remaining myelin debris, the microvessels pellet was recovered and extensively washed in PBS/HEPES.

\subsection{Cell isolation}


Isolated CP and remaining brain tissue were further processed in order to obtain single cell suspensions. CP samples were incubated $\left(20 \mathrm{~min}, 37^{\circ} \mathrm{C}\right)$ in digestion buffer (PBS w/ $\mathrm{Ca} / \mathrm{Mg}, 2 \% \mathrm{v} / \mathrm{v}$ FBS, $10 \mathrm{mM}$ HEPES) containing $1 \mathrm{mg} / \mathrm{ml}$ DNAse I (Sigma, \#DN25) and 1 mg/ml Collagenase/Dispase (Sigma, \#11097113001). Digested tissues were mechanically

155 dissociated using syringes connected to 22- and 26G needles, then cells were washed with

156 FACS buffer, and used for further analysis. For the isolation of brain cells, hemispheres were 157 minced with a scalpel, and homogenized in digestion buffer (HBSS, with $6.75 \mathrm{~g} / 1$ glucose, 20 $158 \mathrm{mM}$ HEPES) containing DNAse I and Collagenase/Dispase as previously mentioned.

159 Homogenate was incubated $\left(40 \mathrm{~min}, 37^{\circ} \mathrm{C}, 200 \mathrm{rpm}\right)$, and filtered through a $70 \mu \mathrm{m}$ cell strainer 160 (Falcon $\left.{ }^{\circledR}, \# 352350\right)$. The cell suspension was centrifuged $\left(400 \mathrm{~g}, 10 \mathrm{~min}, 4{ }^{\circ} \mathrm{C}\right)$, and the cell 161 pellet separated in a $25-70 \%$ discontinuous Percoll® gradient for 20 min without brake. Cells were recovered from the gradient interface, washed with FACS buffer, and used for further analysis.

\subsection{Flow-cytometric analysis}

Cells were resuspended in FACS buffer, and stained as previously described $(39,40)$. All antibodies were purchased from BioLegend unless otherwise stated. In short, cells were

167 incubated with an anti-mouse CD16/32 antibody (clone 93, BioLegend, 101302) and stained 168 with fixable viability dye ZombieNIR, for $20 \mathrm{~min}$ at $4^{\circ} \mathrm{C}$. Subsequently, cells were stained (30 169 min, $4{ }^{\circ} \mathrm{C}$ ) using fluorochrome-conjugated antibodies CD11b (PerCP-Cy5.5) eBioscience, 170 CD45 (BV510), MHCII (BV711), and CD31 (APC) BD Biosciences. Cells were washed

171 (400 g, $5 \mathrm{~min} 4^{\circ} \mathrm{C}$ ) with FACS buffer, fixed in $4 \%$ PFA for $15 \mathrm{~min}$ at $4{ }^{\circ} \mathrm{C}$ and re-suspended in 172 FACS buffer. For CP, cells were additionally permeabilized with eBioscience ${ }^{\mathrm{TM}}$ 173 Permeabilization Buffer (Invitrogen), incubated with antibody rabbit-anti-TTR (Abcam) for 40 174 min, and stained with secondary antibody anti-rabbit AF488 (ThermoFisher). Cells were 
175 acquired using Attune NxT Flow Cytometer (Thermo Fischer). Data was analysed using FlowJo 176 software (version 10.5.3, FlowJo LLC, OR, USA).

177

178

180

181

182

183

184

185

186

187

188

189

190

191

192

193

194

195

196

197

198

\subsection{FITC-dextran permeability assay}

BCSFB and BBB permeability were measured as previously described (34) with modifications. In short, 4 kDa FITC-dextran (Sigma, \#46944) was diluted in PBS, and administered intravenously (i.v.) at $75 \mathrm{mg} / \mathrm{kg}$ body weight mice, $30 \mathrm{~min}$ before CSF and brain collection. CSF was collected through the cisterna magna as previously described, diluted 100fold in PBS, and spun down (1000 g, $5 \mathrm{~min})$. The resulting supernatant were further used for analysis. After perfusion, isolated brains were weighted, minced with a scalpel, homogenized in formamide (Roth) at $0.8 \mathrm{ml}$ per $100 \mathrm{mg}$ tissue, and incubated overnight $\left(37^{\circ} \mathrm{C}, 200 \mathrm{rpm}\right)$. Homogenates were spun down (12,000 g, $5 \mathrm{~min})$, and supernatants were collected and diluted 2-fold in PBS. All samples were measured in triplicates at $\lambda_{\text {ex }} / \lambda_{\text {em }}=485 / 520 \mathrm{~nm}$ using SpectraMaxM5e (Molecular Devices LLC).

\subsection{Choroid plexus epithelial cells and $T$. gondii in vitro infection}

Primary CP epithelial cell culture was obtained as previously described $(43,44)$ with modification. Isolated CP from 9 to 14 mice, one week-old, were used in each preparation. Tissues were pooled in digestion buffer (PBS w/ Ca/Mg, 2\% v/v FBS, $10 \mathrm{mM} \mathrm{HEPES}$ ) containing $1 \mathrm{mg} / \mathrm{ml}$ DNAse I and $2 \mathrm{mg} / \mathrm{ml}$ Collagenase/Dispase, and incubated $\left(20 \mathrm{~min}, 37^{\circ} \mathrm{C}\right)$. Digested tissues were mechanically dissociated and cells were washed (400 g 5 min) with FACS buffer (PBS w/o Ca/Mg, 2mM EDTA, 2\% v/v FBS, $10 \mathrm{mM}$ HEPES). Cell pellet was resuspended, and cultivated with complete medium (DMEM/F12, supplemented with $10 \% \mathrm{FBS}$, 1\% Penicillin/Streptomycin, 1\% ITS (Insulin-Transferrin-Selenite), $40 \mathrm{mg} / \mathrm{ml}$ human-EGF (epidermal growth factor, PeproTech, Germany, \#AF-100-15). Cells were grown until confluence, for approximately 7 to 10 days, in 12 well plates previously coated with poly-L- 
199 lysine (PLL), or on top of PLL coated coverslips. Once confluent, cells were infected at 200 multiplicity of infection (MOI) $=5$ with type II PTG-GFPS65T tachyzoites, and incubated at

$20137^{\circ} \mathrm{C}$. After $6 \mathrm{~h}$, culture medium was removed, cells were washed with PBS, and further used 202 for total RNA/DNA isolation, flow cytometric analysis (validation) or immunofluorescence. 203 Naïve controls were treated with pre-warmed fresh medium.

\subsection{Immunofluorescence}

Immunofluorescence staining was performed for $\mathrm{CP}$ whole mount, BMV mount, brain sections and cell culture coverslips. For brain sections, isolated brains were post-fixed $(4 \mathrm{~h}$, at $4{ }^{\circ} \mathrm{C}$ ), soaked in $30 \%$ sucrose in PBS $\left(2\right.$ days, at $4{ }^{\circ} \mathrm{C}$ ), and frozen in cryo media (OCT Compound, Tissue Tek). Coronal sections $(20 \mu \mathrm{m})$ were obtained (Thermo Scientific CryoStar NX50) and only sections containing $\mathrm{CP}$ were stained. BMV mount, brain sections and coverslips were stained directly on glass slides. CP whole mount staining were performed utilizing a free-floating approach. All samples from different origins were stained and mounted with the same protocol unless otherwise stated. In short, samples were fixed (4\% PFA in PBS, $20 \mathrm{~min}, 4{ }^{\circ} \mathrm{C}$ ), washed twice with washing solution (PBS $0.1 \%(\mathrm{v} / \mathrm{v})$ Triton $\mathrm{X}-100$ ), and blocked/ permeabilized with PBS 0.3\% (v/v) Triton X-100 5\% normal-goat-serum (NGS) and unconjugated $\mathrm{F}\left(\mathrm{ab}^{\prime}\right) 2-$-Goat anti-Mouse $\operatorname{IgG}(\mathrm{H}+\mathrm{L})$ antibody (1:500, Thermo Scientific), for 2 h at $4{ }^{\circ} \mathrm{C}$. Next, samples were incubated with antibody solution (PBS $0.1 \%(\mathrm{v} / \mathrm{v})$ Triton X-100 $2 \%$ NGS) containing the primary antibodies of interest: anti-SAG1 (1:50), anti-GFP (1:1000), anti-E-cadherin (1:500), anti-PDGFR $\beta$ (1:200), anti-ZO-1(1:50), anti-IBA-1(1:500), antiCD31 (1:50), and anti-Claudin-2 (1:500). Primary antibodies were incubated overnight at $4{ }^{\circ} \mathrm{C}$, then samples were washed twice, and incubated (30 min, RT) in antibody solution with secondary antibodies (1:1000) tagged with AF488, AF555, AF594 and AF647. Next, samples were washed twice, and mounted with ProLong ${ }^{\text {TM }}$ Gold Antifade Mountant with DAPI (ThermoFisher). In some samples, anti-CD45 antibody conjugated with AF647 was used, and 
224 an additional final staining step was added. For brain sections, antigen retrieval (10 mM citrate 225 buffer, $\mathrm{pH} 6.0,0.1 \%$ Tween-20) was performed at $96{ }^{\circ} \mathrm{C}$ for $30 \mathrm{~min}$, before the blocking and 226 permeabilization step. Images were generated using a Leica TCS SP8 microscope, and analysed 227 using the ImageJ software (ImageJ 1.52p).

\subsection{DNA and RNA isolation}

Total DNA and RNA were isolated from CP, BMV, spleen, spinal cord, brain and CP epithelial cell culture. Samples from CP, BMV and CP epithelial cell culture were isolated with Quick-DNA/RNA Miniprep kit (Zymo Research, Germany) according to the manufacturer's instructions. Brain, spinal cord, and spleen samples were first homogenized with TriFast (Peqlab, 30-2010) using tubes containing Zirconium oxide beads (Precellys, P000926-LYSK0A) in a BeadBug 6 homogenizer (Biozym). DNA was isolated from the homogenate according to the manufacturer's instructions. RNA was isolated from the homogenate by isopropanol precipitation or using peqGOLD total RNA kit (Peqlab) following the manufacturer's instructions. The concentration and purity of DNA and RNA samples were determined using NanoDrop 2000 spectrophotometer (ThermoFisher; Germany), and samples were stored at -80 ${ }^{\circ} \mathrm{C}$ until further use.

\subsection{2. $q P C R$}

Parasite burden was assessed in triplicates using $40 \mathrm{ng}$ of isolated DNA, FastStart

242 Essential DNA Green Master and LightCycler ${ }^{\circledR} 96$ System (both Roche, Germany), as previously described (40). Thermal-cycling parameters were set as follows: initial activation $\left(95^{\circ} \mathrm{C}, 10 \mathrm{~min}\right), 55$ amplification cycles consisting of denaturation $\left(95^{\circ} \mathrm{C}, 15 \mathrm{~s}\right)$, annealing $(60$ ${ }^{\circ} \mathrm{C}, 15 \mathrm{~s}$ ) and elongation $\left(72{ }^{\circ} \mathrm{C}, 15 \mathrm{~s}\right)$. The DNA target was the published sequence of the highly conserved 35-fold-repetitive B1 gene of $T$. gondii $(45,46)$. Murine argininosuccinate lyase $(A s l)$ was used as reference gene for normalization and relative DNA levels were determined by the 
ratio gene of interest / reference gene and subsequently normalized to mean values of control group (47). Primers were synthetized by Tib MolBiol (Germany) and used at $200 \mathrm{nM}$ final concentration. Primer sequences are described elsewhere [see Additional file 1].

\subsection{RT-qPCR} junctions and MMPs were assessed in triplicates using 20 ng total RNA, TaqMan® RNA-to$\mathrm{C}_{\mathrm{T}}^{\mathrm{TM}}$ 1-Step Kit (Applied Biosystems, Germany) and LightCycler ${ }^{\circledR} 96$ (Roche, Germany) as previously described (40). Thermal-cycling parameters were set as follows: reverse transcription $\left(48{ }^{\circ} \mathrm{C}, 30 \mathrm{~min}\right)$, inactivation $\left(95{ }^{\circ} \mathrm{C}, 10 \mathrm{~min}\right)$ followed by 55 cycles of denaturation $\left(95^{\circ} \mathrm{C}, 15 \mathrm{~s}\right)$ and annealing/extension $\left(60{ }^{\circ} \mathrm{C}, 1 \mathrm{~min}\right)$. Utilized TaqMan ${ }^{\circledR}$ Gene Expression Assays (Applied Biosystems, Germany) are listed elsewhere [see Additional file 1]. Hprt was chosen as reference gene and relative mRNA levels were determined by the ratio gene of interest / reference gene and subsequently normalized to mean values of control group.

262 CT ${ }^{\mathrm{TM}}$ 1-Step Kit (Applied Biosystems, Germany) was used. Samples were analyzed in 263 triplicates (20 ng of isolated mRNA per reaction) using LightCycler® 96 with the following 264 parameters: reverse transcription $\left(48^{\circ} \mathrm{C}, 30 \mathrm{~min}\right)$, inactivation $\left(95^{\circ} \mathrm{C}, 10 \mathrm{~min}\right)$ followed by 55 cycles of denaturation $\left(95^{\circ} \mathrm{C}, 15 \mathrm{~s}\right)$ and annealing/extension $\left(60^{\circ} \mathrm{C}, 1 \mathrm{~min}\right)$ and melting curve

266 analysis. The primer sequences are listed elsewhere [see Additional file 1] and were synthetized 267 by Tib MolBiol and used at $100 \mathrm{nM}$ final concentration. Hprt was chosen as reference gene and 268 relative mRNA levels were determined by the ratio gene of interest / reference gene and 269 subsequently normalized to mean values of control group.

\subsection{Statistical analysis}


Results were statistically analyzed using GraphPad Prism 7 (GraphPad Software Inc.,

272 USA), post-test corrections were applied according to software recommendations. Statistical significance was set to $p \leq 0.05$. All data are presented as arithmetic mean and standard error of the mean (SEM) and are representative of at least two independent experiments. For parasite burden analysis, normalized data were analyzed by multiple $t$-test, with Holm-Šidák correction for multiple comparisons. For flow cytometric analyses, normalized data were analyzed by oneway ANOVA, with Tukey's correction. For RT-qPCR, data were analyzed by one-way ANOVA followed by Dunnett's correction. BCSFB permeability assay was analyzed by multiple $t$-test to compare between BCSFB and BBB, and one-way ANOVA followed by Dunnett's correction was used to compare differences between day 0 and the time-point analyzed from the same barrier type. RT-qPCR data from primary cell culture was analyzed by student $t$-test.

\section{Results}

\subsection{Choroid plexus is infected by $T$. gondii upon the invasion}

BCSFB is a gateway to the CNS for different pathogens as well as immune cells under certain inflammatory conditions $(26,35,48-50)$. Assuming that the $\mathrm{CP}$ is involved in the

287 parasite entry and early immune response upon the invasion of the brain by $T$. gondii, we investigated the presence of parasites in the CNS through the course of infection. As BBB endothelial cells are cellular targets for T. gondii (7), we isolated brain microvessels (BMV) and CP Fig1A to assess parasite burden over time by PCR. Our results revealed the early presence of parasites on $\mathrm{CP}$ at 3 dpi (3 days post-infection), which was increased by day 5 and

292 reduced by day 7 Fig1B. In the BMV, the parasite burden only strongly increased by day 7

293 Fig1B. Next, CP and BMV of infected animals were isolated and immuno-stained as a threedimensional whole mount preparation. Within the $\mathrm{CP}$ at $5 \mathrm{dpi}$, we detected parasites $\left(\mathrm{SAG}^{+}\right)$ 
stromal regions between the CP epithelial cells $\left(\right.$ E-cadherin $\left.^{+}\right)$without co-localization with recruited $\mathrm{CD} 45^{+}$cells Fig1C. In the BMV, parasites were found to be associated to PDGFR $\beta^{+}$ pericytes and ZO- $1^{+}$endothelial cells Fig1D, which constitute the main components of cerebral microvasculature forming the BBB (51). Overall, parasites were found in the $\mathrm{CP}$, and apparently more frequently in initial infection at the BCSFB interface than the BBB. Immunostaining of isolated BMV confirmed the presence of $T$. gondii tachyzoites in BMV at $7 \mathrm{dpi}$, as detected previously by PCR [see Additional file 2]. Spleens were used as controls to monitor the parasite burden levels in the periphery, indicating the usual spread of the parasites throughout tissues [see Additional file 2]. Parasite burden was just detected starting at 7dpi in both brain parenchyma (after $\mathrm{CP}$ removal) and spinal cord, evidencing the early infection through the CP and the microvasculature [see Additional file 2].

\subsection{Choroid plexus endothelial and immune cells are targeted by $T$. gondii}

Given the early presence of $T$. gondii in the CP, we sought to determine the target cell types in this compartment. Therefore, mice were infected i.p. with $10 \mathrm{e} 5$ tachyzoites of the $T$. gondii strain expressing the fluorescent protein GFP (PTG-GFPS65T). First, we analyzed coronal brain sections showing $\mathrm{CP}$ from lateral ventricles and found parasites within the choroidal endothelial cells at 7dpi Fig2A [and see Additional file 3]. Indeed, at 7dpi parasites were additionally found in the cortex and the adjacent brain areas [see Additional file 2], simultaneously to the CP [see Additional file 3]. To identify infected immune cells in the BCSFB, isolated CP whole mounts were co-stained for CD45 and CD31. At 3dpi, we observed T. gondii parasites associated to immune cells and endothelial cells in the CP Fig2B. Of note, recruited amoeboid-shape $\mathrm{CD} 45^{\text {hi }}$ leukocytes did not appear to be associated to parasites, rather a resident stellate $\mathrm{CD} 45^{\text {int }} \mathrm{CP}$ macrophages (referred as CP-BAMs (border-associatedmacrophages) or epiplexus cells) were co-localized with parasites at this time point [see Additional file 3]. CP endothelial cells were infected with intracellular parasites at 3 and 5dpi, but at 7dpi infected cells were less abundant. Additionally, parasites were also found in the 
322 inner core of the CP parenchyma, suggesting the infection of mesenchymal derived stromal

323 cells. Altogether, our results demonstrate the dynamic infiltration of parasites into endothelial

324 and immune cells of the CP in the early phase of infection, confirming our initial data on 325 parasite burden.

\subsection{Early immune response at the choroid plexus prior to the $B B B$}

Pathogens infecting the $\mathrm{CP}$ elicit local immune responses which are associated with the activation of endothelial, epithelial and tissue-resident immune cells (35). To characterize the early immune response to $T$. gondii in the $\mathrm{CP}$ in more detail, we first compared the MHCII expression on endothelial cells from $\mathrm{CP}$ and remaining brain tissue Fig3A-H. In agreement with the spatiotemporal differences in pathogen distribution Fig1, MHCII upregulation on endothelial cells was first evidenced in the $\mathrm{CP}$ followed by later activation of brain endothelial cells Fig3A-H. The kinetics of MHCII expression by microglia was comparable to brain endothelial cells and reached maximum levels at 7dpi [see Additional file 4]. Moreover, MHCII expression was analyzed on $\mathrm{CP}$ epithelial cells even though less than $10 \%$ of the cells express this marker, a significant increase was observed [see Additional file 4]. Of note, total mRNA expression of MHCII $(\mathrm{H} 2-\mathrm{Aa})$ in the $\mathrm{CP}$ was also increased during infection [see Additional file 5]. Taken together, choroidal endothelial cells responded earlier to $T$. gondii than brain endothelial cells and microglia, indicating that the $\mathrm{CP}$ is a critical interface for early parasite recognition.

To further compare the local inflammatory response in the BCSFB versus the BBB, we analyzed the mRNA expression of pro-inflammatory cytokines which play a central role in host

343 defense upon T. gondii infection. Therefore, CP and the BMV were isolated from infected 344 brains and expression of IFN- $\gamma$, TNF, and IL-6 were measured Fig3I. Compared to the BMV, 345 CP expression of IFN- $\gamma$ was higher and earlier elevated. BMV only reached comparable IFN- $\gamma$ 346 levels at 7dpi. TNF was significantly higher in the CP at both 5 and 7dpi and IL-6 levels peaked 347 at $3 \mathrm{dpi}$ and was higher in the $\mathrm{CP}$ at $7 \mathrm{dpi}$ Fig3I. We also evaluated the expression of type I 
348 interferon- $\beta$ (IFN- $\beta$, Ifnb1), which were highly expressed in the CP [see Additional file 5].

349 Furthermore, the cell-autonomous immune response against intracellular T. gondii infection is

350 mediated by IFN- $\gamma$-inducible GTPases, designated as host-defense-factors (Irgm1, Igtp,

$351 G b p 2 b)$. Following the elevated IFN- $\gamma$ levels, we detected higher expression of those genes on

352 the CP compared to the BMV Fig3J. Previous studies have shown specific immune cell

353 trafficking molecules to be up-regulated on CP epithelial cells in the presence of IFN- $\gamma$ and

354 TNF (50). Accordingly, CXCL9 expression was higher in CP throughout the observation

355 period. On the contrary CXCL10 was more pronounced in the BMV, and both chemokines were

356 progressively increased during the infection Fig3K. At last, M-CSF (macrophage colony-

357 stimulating factor, Csfl) and CX3CL1 (fractalkine), which are chemokines involved in

358 monocyte trafficking across the BCSFB (52) were also found up-regulated in the CP at 7dpi,

359 while no alteration was detected for M-CSF on BMV [see Additional file 5]. Of note, ICAM-1

360 involved in the general trafficking of immune cells was also up-regulated at the CP [see

361 Additional file 5]. Overall, T. gondii elicited an early and local immune response at the BCSFB,

362 with the expression of pro-inflammatory cytokines, IFN- $\gamma$-inducible GTPases, and leukocyte

363 trafficking molecules.

$364 \quad$ 3.4. Loss of $\mathbf{T J}$ integrity affects BCSFB function in early infection

TJ molecules constitute the main components of the paracellular barrier established by

$\mathrm{CP}$ epithelial cells, therefore they assume an essential role determining cellular and molecular

367 compounds delivered from blood into CNS (25). To investigate the outcome of T. gondii

368 infection at the BCSFB, we analyzed the expression of TJs in isolated CP tissue over the course

369 of infection. Claudins form the majority group of the integral membrane TJ proteins, which

370 establish complex interactions with intracellular linker proteins like zonula occludens proteins

371 (ZO, Tjp) (53). Upon T. gondii infection at the CP, we detected lower expression levels of

372 Claudin-2, -3, -5, and -11 compared to naïve group, suggesting a TJ alteration Fig4A. 
373 Moreover, the breakdown of epithelial barriers has been associated with increased activity of matrix metalloproteinases (MMPs), which were indicated to be detrimental for BCSFB

375 integrity during neuroinflammation (33). Here, infected CP tissue displayed a robust up376 regulation of MMP-8 and MMP-13 Fig4B, pointing towards a potential involvement of those 377 endopeptidases in the epithelial barrier perturbation upon parasite invasion of the BCSFB.

378 Taken together, the expression levels of claudins and MMPs in the infected CP suggested a 379 barrier disruption. Indeed, immunostaining for claudin-2 on brain sections showed ruffled380 shape irregularities (white arrows) on the contour of CP epithelial cells in infected mice, instead 381 of the smooth and continuous staining pattern depicted in naïve CP Fig4C. Finally, we 382 determined whether altered TJ and MMP expression patterns affected CP barrier function. For 383 this purpose, a FITC-dextran permeability assay was performed to assess barrier function in 384 vivo. The extravasation of the fluorochrome-conjugated polysaccharide was quantified in the

385 CSF for CP integrity, and in the remaining brain homogenate for BBB integrity Fig4D. In 386 contrast to the BBB, BCSFB permeability increased over time. A significant difference between 387 both compartments was first visible at 7dpi and further increased from 10dpi to $23 \mathrm{dpi}$. Thus, 388 our results reveal a continuous increase in BCSFB permeability, suggesting long-standing 389 detrimental effects of T. gondii infection on CP barrier integrity. Altogether, T. gondii infection 390 results in TJs disturbances in the CP, with possible involvement of MMP-8 and MMP-13, 391 culminating in functional loss and increased permeability of the BCSFB.

\subsection{Response of choroid plexus epithelial cells to $T$. gondii in vitro}

For a more detailed characterization of $T$. gondii infection effects in the CP epithelium,

394 we developed an in vitro culture model for murine primary CP epithelial cells. First, we 395 confirmed the ability of the cultivated cells to establish adhesion junctions and express ZO-1 396 (honeycomb shape) Fig5A. In addition, cell cultures were largely devoid of IBA- $1^{+}$myeloid 397 cells and contained mainly $\mathrm{CD}^{-} 5^{-} \mathrm{CD}^{-} 1^{-}$E-cadherin ${ }^{+}$epithelial cells Fig5B. Subsequently, CP 
epithelial cells were cultured in the presence or absence of $\mathrm{GFP}^{+} T$. gondii and the expression of the TJ proteins ZO-1 and claudin-2 were quantified. ZO-1 Fig5C and Claudin-2 Fig5D showed a poor and non-continuous distribution, characterized by strand breaks and puncta upon infection, compared to naïve controls. Additionally, the gene expression analysis of the 402 cultivated epithelial cells showed no expression of IFN- $\gamma$, but increased expression of IFN- $\gamma-$ 403 receptor (Ifngr2) upon infection. The tissue damage-associated cytokines TNF and IL-6 were also upregulated, and the chemokine CCL2, responsible for myeloid cell recruitment was about 60-fold higher on infected cultures Fig5E. We also detected a discrete reduction of ZO-1 (encoded by Tjp1) expression upon infection, without change and in the levels of claudin-2 and claudin-11 Fig5F. In agreement with Fig4B, MMP-8 and -13 showed a robust upregulation 408 upon infection, suggesting that CP epithelial cells are relevant source of MMPs Fig5G. Overall, these results demonstrate that epithelial cells from CP directly respond to $T$. gondii infection, and contribute to the local inflammatory response and BCSFB damage.

\section{Discussion} the recent years $(49,54-56)$, and distinct pathogens have been described exploiting the CP as

414 gateway into CNS (35). These microbes cross CNS barriers via paracellular entry, transcellular 415 penetration, or via infected immune cells ("Trojan horse") (57). The same mechanisms have 416 been proposed for $T$. gondii invasion of the BBB (23), but only few studies have explored the 417 CP as a gateway for T. gondii (36).

Here we demonstrate that $T$. gondii was found in the $\mathrm{CP}$ at the onset of infection. In a 419 model of reactivated TE, there was no evidence for the involvement of the CP in $T$. gondii 420 dissemination $(37,38)$. Whether parasites are able to translocate from blood to the CSF, via CP, 421 and further infect CSF-inundated areas (e.g. brain, spinal cord) is still not fully understood. In 422 fact, we have previously described the extent of spinal cord pathology in a model of 
experimental chronic toxoplasmosis, suggesting that parasites translocate into CSF while the infection progresses (58). Currently, the lack of tools to specifically block parasite entry into one or another barrier presents a major challenge to unveil their respective contributions to the parasite invasion. Here, we propose that $\mathrm{CP}$ infection is the initial step to the development of

427 the neuroinflammation, resulting in the disruption of the BCSFB. Evidences show that the 428 collapse of this barrier and its regulatory mechanisms allow immune cells to enter the CNS and initiate neuroinflammatory diseases (27). As the disease evolves, the parasite spread increases e.g. through circulation of infected monocytes which simultaneously can cross the CNS via BBB and CP transposition. It is likely that the higher parasite burden on BMV at 7dpi indicate infected embolized monocytes in small CNS capillaries, as suggested by previous studies (59). Our findings suggest that the activation of the $\mathrm{CP}$ vasculature is involved in the orchestration of the anti-parasitic immune responses at early time points of infection. We observed that choroidal endothelial cells upregulated MHCII expression in response to T. gondii earlier than brain endothelial cells and microglia. Of note, brain endothelial cells have been described to serve as a replicative niche for $T$. gondii invasion of the CNS (7), but whether activated endothelial cells can control parasite replication in vivo is still unknown. Vascular and epithelial alterations at the $\mathrm{CP}$, followed by other alterations like reduced choroidal synthesis, transport capacity, and CSF secretion were shown during aging, and were described to be intensified in Alzheimer's disease (60). Our data confirm the CP as an important interface to 442 early pathogen interaction with endothelial cells, likely promoting damage recognition and 443 antigen-presentation.

Infections at the BCSFB has proven to promote CNS inflammation, inducing cytokines and chemokine secretion locally, and in the CSF, mediating immune cell activation and recruitment (61). The CP senses CNS injury and rapidly responds to inflammation, upregulating

447 the expression of adhesion molecules and chemokines receptors essential for leukocyte 448 trafficking (50). Upon the establishment of infection at the CP, we have found that $T$. gondii 
elicited early and local immune response at the BCSFB, with expression of pro-inflammatory cytokines, IFN- $\gamma$-inducible GTPases, and leukocyte trafficking molecules. In face of early detection of $\mathrm{CD}_{4} 5^{+}$cells associated to $T$. gondii on the $\mathrm{CP}$, we hypothesize that a transient burden of extracellular parasites in the blood likely reach the $\mathrm{CP}$ interface, infecting the choroidal endothelial cells and challenging the resident stromal macrophages and epithelial cells. Additionally, IFN- $\gamma$, TNF and IL-6 upregulation in the CP of infected mice might be due to the combined action of immune and epithelial cells, which showed elevated levels of gene expression after in vitro culture with $T$. gondii. This suggests a direct anti-parasitic, proinflammatory response of the CP epithelial cells. However, IFN- $\gamma$ gene expression was only detectable in the CP in vivo but not in isolated CP epithelial cells co-cultured with $T$. gondii. This indicates that IFN- $\gamma$ expression is restricted to immune cells. Whether they are CP-resident and/or recruited immune cells from the periphery remains to be clarified. IFN- $\gamma$-signaling through CP epithelium have been shown to tightly regulate cell recruitment into CNS (50), and probably will determine the immune cell dynamics through the CP upon $T$. gondii infection. Besides, we detected upregulation of IFN- $\gamma$-receptor (Ifngr2) on in vitro infected CP epithelial 464 cells, likely resulting in the increased expression of leukocyte trafficking genes CSF-1, 465 CX3CL1, ICAM-1, CXCL9 and CXCL10, as previous described (50). Moreover, we found CXCL9 more expressed in CP rather than in BMV through the first week of infection. Indeed, CXCL9 is known to induce T-cell activation and recruitment into the brain during cerebral toxoplasmosis (62). Hereby, CXCL9 could be a differential trafficking molecule expressed by the $\mathrm{CP}$ to specific modulated the control of $T$. gondii infection.

Dysfunctional BCSFB is part of the pathophysiology leading to increased neuroinflammation (32). At the BCSFB, the permeability is determined by TJ, specifically the

472 claudin-family proteins. Claudin-1, $-2,-3$ are highly expressed in the $\mathrm{CP}$, and have been shown 473 to be sensitive to inflammation (63-66). In our analysis, claudin-2 was downregulated and 474 overall structurally disorganized within CP epithelial cells, displaying a ruffled shape upon 
475 infection. TJ ruffling frequently correlates with increased paracellular permeability caused by 476 altered anchoring into actin filaments (67-69). This anchoring is dependent on the scaffold

477 protein ZO-1, here, also shown to be changed in the infected CP. Accordingly, alterations of 478 claudins and $\mathrm{ZO}-1$ in the $\mathrm{CP}$ have also been described in experimental autoimmune 479 encephalomyelitis (EAE) and LPS- sepsis models $(70,71)$. Of note, the infection of BCSFB by 480 Trypanosoma brucei has indicated direct parasite interactions with claudins of the CP, although 481 the mechanisms of how they induce TJs opening is still not known $(72,73)$. Still, the disruption 482 of the TJ has been associated to the activity of matrix metalloproteinases (MMP), enzymes 483 implicated on BCSFB breakdown in neurodegenerative diseases and bacterial meningitis (29, 484 74). We detected increased expression levels of the MMP-8 and MMP-13 on the infected CP, 485 with both MMP upregulated on in vitro CP epithelial cultures after T. gondii infection. Indeed, 486 previous studies have described MMP-8 contribution to BCSFB leakage, and MMP-13 association with $\mathrm{TJ}$ dysregulation $(31,75)$.

Importantly, here we discovered that the CP damage extended to the chronic phase of experimental murine toxoplasmosis, presented by increased BCSFB permeability, which 490 correlates to our previous findings on neuronal impairment during chronic toxoplasmosis (18, $49120,39,76)$. Therefore, we propose that the substantial change on the CP permeability has

492 detrimental implications for CSF composition and impairment of the CNS drainage. Both 493 aspects may contribute to the increased neuroinflammation and subsequent neuronal damage 494 during toxoplasmosis.

\section{5. Conclusion}

In summary, we detected that the $\mathrm{CP}$ is initially infected early after the onset of $T$. gondii

497 infection, where next to $\mathrm{CP}$ endothelial cells also immune cells are targeted, determining a rapid 498 inflammatory response and loss of BCSFB integrity and functionality. These processes are

499 likely driven by TJ disturbance of claudins within CP epithelial cells and by MMP activity. 
500 Thus, dysfunctional BCSFB may early enhance and further contribute to T. gondii-induced

501 neuroinflammation, therefore emerging as a crucial target for new therapeutic approaches in

502 cerebral infections.

503 6. Abbreviations

504 AF488 - Alexa Fluor 488

505 AF555 - Alexa Fluor 555

506 AF594 - Alexa Fluor 594

507 AF647 - Alexa Fluor 647

508 ANOVA - analysis of variance

509 BBB - blood-brain barrier

510 BCSFB - blood-cerebrospinal fluid barrier

511 BMV - brain microvessels

512 CCL2 - chemokine (C-C motif) ligand 2

513 CD - cluster of differentiation

514 CNS - central nervous system

515 CP - choroid plexus

516 CP-BAMS - choroid plexus border-associated macrophages

517 CSF - cerebrospinal fluid

518 CX3CL1 - chemokine (C-X3-C motif) ligand 1

519 CXCL10 - chemokine (C-X-C motif) ligand 10

520 CXCL9 - chemokine (C-X-C motif) ligand 9

521 DAAD - Deutscher Akademischer Austauschdienst

522 DAPI - 4',6-diamidino-2-phenylindole

523 DFG - Deutsche Forschungsgemeinschaft

524 DMEM - Dulbecco's Modified Eagle Medium

525 DNA - deoxyribonucleic acid

526 DNAse I - deoxyribonuclease type I

527 dpi - days post infection

528 EDTA - ethylenediamine tetraacetic acid

529 EGF - epidermal growth factor

$530 \quad$ FACS - fluorescence-activated cell sorting

531 FBS - fetal bovine serum

532 FITC - fluorescein isothiocyanate

$533 \mathrm{Gbp} 2 \mathrm{~b}$ - Guanylate binding protein 1

534 GFP - green fluorescent protein

535 HBSS - Hank's balanced salt solution

536 HEPES - 4-(2-hydroxyethyl)-1-piperazineethanesulfonic acid

537 HFF - human foreskin fibroblasts

538 IBA-1 - ionized calcium binding adaptor molecule 1

539 ICAM-1 - Intercellular Adhesion Molecule 1 
$540 \quad$ Ifngr2 - IFN- $\gamma$-receptor

$541 \quad$ IFN- $\beta$ - type I interferon-beta

$542 \quad$ IFN- $\gamma-$ interferon gamma

$543 \quad$ IgG - immunoglobulin G

544 Igtp - Interferon gamma-induced GTPase

545 IL-6 - interleukin 6

546 Irgm 1 - Immunity-related GTPase family M protein 1

547 ITS - insulin-transferrin-selenite

548 LPS - Lipopolysaccharides

549 M-CSF - macrophage colony-stimulating factor

550 MHCII - major histocompatibility complex type II

551 MMPs - metalloproteinases

552 MOI - multiplicity of infection

553 NEEA - non-essential amino acids

554 NGS - normal goat serum

555 OCT - optimal cutting temperature

556 PBS - phosphate-buffered saline

557 PCR - polymerase chain reaction

558 PDGFR $\beta$ - platelet derived growth factor receptor $\beta$

559 PFA - paraformaldehyde

560 PLL - poly-L-lysine

561 RNA - ribonucleic acid

562 RT - room temperature

563 RT-qPCR - Quantitative reverse transcription PCR

564 SAG1 - surface antigen 1

565 SEM - standard error of the mean

566 T.gondii -Toxoplasma gondii

567 TE - toxoplasma encephalitis

568 Tjp1 - tight junction protein-1

569 TJs - tight junctions

570 TNF - tumor necrosis factor

571 TTR - transthyretin

572 ZO-1 - zona occludens-1

$573 \quad 7 . \quad$ Declarations

$574 \quad$ 7.1. Ethical approval

All animal experiments were approved by the respective authorities

576 (Landesverwaltungsamt Halle, Sachsen-Anhalt, Germany) in accordance with German and

577 European legislation. 


\subsection{Consent for publication}

579 Not applicable

\subsection{Availability of data and materials}

581 The datasets used and/or analyzed during the current study are available from the corresponding

582 author on reasonable request.

\subsection{Competing interests}

584 The authors declare no competing interests.

\subsection{Funding}

586 This work was supported by the DFG SFB 854 and SPP 1937 to IRD, by the DAAD research

587 grant for doctoral programmes in Germany ( $\left.{ }^{\circ} 57214224\right)$ and by the Promotionsstipendium of 588 the Medizinischen Fakultät Magdeburg to CF.

\section{7.6. Authors' contributions}

590 CAF and SA performed experiments and analyzed data. JS, LM, AK, OL, MAD, TD critically 591 discussed experimental design, provided material and co-edited the manuscript. CAF and IRD 592 conceived experimental design, and wrote the manuscript.

\section{$593 \quad$ 7.7. Acknowledgments}

594 We thank Petra Grüneberg and Dr. Abidat Schneider for the excellent technical assistance, and 595 all lab colleagues indirectly involved on the execution of this work.

596 8. References

597 1. Tenter AM, Heckeroth AR, Weiss LM. Toxoplasma gondii: from animals to humans. 598 Int J Parasitol. 2000;30(12-13):1217-58. 
599 2. Montoya JG, Liesenfeld O. Toxoplasmosis. Lancet. 2004;363(9425):1965-76.

600 3. Wilking H, Thamm M, Stark K, Aebischer T, Seeber F. Prevalence, incidence 601 estimations, and risk factors of Toxoplasma gondii infection in Germany: a representative, 602 cross-sectional, serological study. Sci Rep. 2016;6:22551.

603 4. Harker KS, Ueno N, Lodoen MB. Toxoplasma gondii dissemination: a parasite's 604 journey through the infected host. Parasite Immunol. 2015;37(3):141-9.

605 5. Barragan A, Sibley LD. Transepithelial migration of Toxoplasma gondii is linked to 606 parasite motility and virulence. J Exp Med. 2002;195(12):1625-33.

607 6. Courret N, Darche S, Sonigo P, Milon G, Buzoni-Gatel D, Tardieux I. CD11c- and 608 CD11b-expressing mouse leukocytes transport single Toxoplasma gondii tachyzoites to the 609 brain. Blood. 2006;107(1):309-16.

6107 Konradt C, Ueno N, Christian DA, Delong JH, Pritchard GH, Herz J, et al. Endothelial 611 cells are a replicative niche for entry of Toxoplasma gondii to the central nervous system. Nat 612 Microbiol. 2016;1:16001.

613 8. Lachenmaier SM, Deli MA, Meissner M, Liesenfeld O. Intracellular transport of 614 Toxoplasma gondii through the blood-brain barrier. J Neuroimmunol. 2011;232(1-2):119-30.

$6159 . \quad$ Ueno N, Lodoen MB, Hickey GL, Robey EA, Coombes JL. Toxoplasma gondii616 infected natural killer cells display a hypermotility phenotype in vivo. Immunol Cell Biol. 617 2015;93(5):508-13.

618 10. Coombes JL, Charsar BA, Han SJ, Halkias J, Chan SW, Koshy AA, et al. Motile 619 invaded neutrophils in the small intestine of Toxoplasma gondii-infected mice reveal a 620 potential mechanism for parasite spread. Proc Natl Acad Sci U S A. 2013;110(21):E1913-22.

621 11. Bhandage AK, Olivera GC, Kanatani S, Thompson E, Lore K, Varas-Godoy M, et al.

622 A motogenic GABAergic system of mononuclear phagocytes facilitates dissemination of 623 coccidian parasites. Elife. 2020;9.

624 12. Olafsson EB, Barragan A. The unicellular eukaryotic parasite Toxoplasma gondii 625 hijacks the migration machinery of mononuclear phagocytes to promote its dissemination. 626 Biol Cell. 2020.

627 13. Ferguson DJ, Graham DI, Hutchison WM. Pathological changes in the brains of mice 628 infected with Toxoplasma gondii: a histological, immunocytochemical and ultrastructural 629 study. Int J Exp Pathol. 1991;72(4):463-74.

630 14. Dubey JP, Lindsay DS, Speer CA. Structures of Toxoplasma gondii tachyzoites, 631 bradyzoites, and sporozoites and biology and development of tissue cysts. Clin Microbiol 632 Rev. 1998;11(2):267-99.

633 15. Dubey JP. History of the discovery of the life cycle of Toxoplasma gondii. Int J 634 Parasitol. 2009;39(8):877-82.

635 16. Parlog A, Harsan LA, Zagrebelsky M, Weller M, von Elverfeldt D, Mawrin C, et al. 636 Chronic murine toxoplasmosis is defined by subtle changes in neuronal connectivity. Dis 637 Model Mech. 2014;7(4):459-69. 
638 17. Blanchard N, Dunay IR, Schluter D. Persistence of Toxoplasma gondii in the central

639 nervous system: a fine-tuned balance between the parasite, the brain and the immune system.

640 Parasite Immunol. 2015;37(3):150-8.

641 18. Lang D, Schott BH, van Ham M, Morton L, Kulikovskaja L, Herrera-Molina R, et al.

642 Chronic Toxoplasma infection is associated with distinct alterations in the synaptic protein

643 composition. J Neuroinflammation. 2018;15(1):216.

644 19. Burgdorf KS, Trabjerg BB, Pedersen MG, Nissen J, Banasik K, Pedersen OB, et al.

645 Large-scale study of Toxoplasma and Cytomegalovirus shows an association between

646 infection and serious psychiatric disorders. Brain Behav Immun. 2019;79:152-8.

647 20. Parlog A, Schluter D, Dunay IR. Toxoplasma gondii-induced neuronal alterations.

648 Parasite Immunol. 2015;37(3):159-70.

649 21. Munoz M, Liesenfeld O, Heimesaat MM. Immunology of Toxoplasma gondii.

650 Immunol Rev. 2011;240(1):269-85.

651 22. Matta SK, Rinkenberger N, Dunay IR, Sibley LD. Toxoplasma gondii infection and its

652 implications within the central nervous system. Nat Rev Microbiol. 2021.

653 23. Mendez OA, Koshy AA. Toxoplasma gondii: Entry, association, and physiological

654 influence on the central nervous system. PLoS Pathog. 2017;13(7):e1006351.

655 24. Ghersi-Egea JF, Strazielle N, Catala M, Silva-Vargas V, Doetsch F, Engelhardt B.

656 Molecular anatomy and functions of the choroidal blood-cerebrospinal fluid barrier in health

657 and disease. Acta Neuropathol. 2018;135(3):337-61.

658 25. Castro Dias M, Mapunda JA, Vladymyrov M, Engelhardt B. Structure and Junctional

659 Complexes of Endothelial, Epithelial and Glial Brain Barriers. Int J Mol Sci. 2019;20(21).

660 26. Baruch K, Schwartz M. CNS-specific T cells shape brain function via the choroid 661 plexus. Brain Behav Immun. 2013;34:11-6.

662 27. Fleischer V, Gonzalez-Escamilla G, Ciolac D, Albrecht P, Kury P, Gruchot J, et al. 663 Translational value of choroid plexus imaging for tracking neuroinflammation in mice and 664 humans. Proc Natl Acad Sci U S A. 2021;118(36).

665 28. Engelhardt B, Sorokin L. The blood-brain and the blood-cerebrospinal fluid barriers: 666 function and dysfunction. Semin Immunopathol. 2009;31(4):497-511.

667 29. Rosenberg GA. Matrix metalloproteinases and their multiple roles in

668 neurodegenerative diseases. Lancet Neurol. 2009;8(2):205-16.

669 30. Vandenbroucke RE. A Hidden Epithelial Barrier in the Brain with a Central Role in 670 Regulating Brain Homeostasis. Implications for Aging. Ann Am Thorac Soc. 2016;13 Suppl 671 5:S407-S10.

672 31. Vandenbroucke RE, Dejonckheere E, Van Lint P, Demeestere D, Van Wonterghem E, 673 Vanlaere I, et al. Matrix metalloprotease 8-dependent extracellular matrix cleavage at the 674 blood-CSF barrier contributes to lethality during systemic inflammatory diseases. J Neurosci. 675 2012;32(29):9805-16. 
676 32. Demeestere D, Libert C, Vandenbroucke RE. Therapeutic implications of the choroid 677 plexus-cerebrospinal fluid interface in neuropsychiatric disorders. Brain Behav Immun.

$678 \quad 2015 ; 50: 1-13$.

679 33. Brkic M, Balusu S, Van Wonterghem E, Gorle N, Benilova I, Kremer A, et al. 680 Amyloid beta Oligomers Disrupt Blood-CSF Barrier Integrity by Activating Matrix 681 Metalloproteinases. J Neurosci. 2015;35(37):12766-78.

682 34. Gorle N, Blaecher C, Bauwens E, Vandendriessche C, Balusu S, Vandewalle J, et al. 683 The choroid plexus epithelium as a novel player in the stomach-brain axis during 684 Helicobacter infection. Brain Behav Immun. 2018;69:35-47.

685 35. Lauer AN, Tenenbaum T, Schroten H, Schwerk C. The diverse cellular responses of 686 the choroid plexus during infection of the central nervous system. Am J Physiol Cell Physiol. 687 2018;314(2):C152-C65.

688 36. Falangola MF, Petito CK. Choroid plexus infection in cerebral toxoplasmosis in AIDS 689 patients. Neurology. 1993;43(10):2035-40.

690 37. Dellacasa-Lindberg I, Hitziger N, Barragan A. Localized recrudescence of

691 Toxoplasma infections in the central nervous system of immunocompromised mice assessed 692 by in vivo bioluminescence imaging. Microbes Infect. 2007;9(11):1291-8.

693 38. Schluter D, Barragan A. Advances and Challenges in Understanding Cerebral 694 Toxoplasmosis. Front Immunol. 2019;10:242.

695 39. Dusedau HP, Kleveman J, Figueiredo CA, Biswas A, Steffen J, Kliche S, et al. $696 \mathrm{p} 75$ (NTR) regulates brain mononuclear cell function and neuronal structure in Toxoplasma 697 infection-induced neuroinflammation. Glia. 2019;67(1):193-211.

698 40. Figueiredo CA, Dusedau HP, Steffen J, Gupta N, Dunay MP, Toth GK, et al. 699 Immunomodulatory Effects of the Neuropeptide Pituitary Adenylate Cyclase-Activating 700 Polypeptide in Acute Toxoplasmosis. Front Cell Infect Microbiol. 2019;9:154.

701 41. Liu L, Duff K. A technique for serial collection of cerebrospinal fluid from the 702 cisterna magna in mouse. J Vis Exp. 2008(21).

703 42. Nakagawa S, Deli MA, Kawaguchi H, Shimizudani T, Shimono T, Kittel A, et al. A 704 new blood-brain barrier model using primary rat brain endothelial cells, pericytes and 705 astrocytes. Neurochem Int. 2009;54(3-4):253-63.

706 43. Menheniott TR, Charalambous M, Ward A. Derivation of primary choroid plexus 707 epithelial cells from the mouse. Methods Mol Biol. 2010;633:207-20.

708 44. Lazarevic I, Engelhardt B. Modeling immune functions of the mouse blood709 cerebrospinal fluid barrier in vitro: primary rather than immortalized mouse choroid plexus 710 epithelial cells are suited to study immune cell migration across this brain barrier. Fluids 711 Barriers CNS. 2016;13:2.

712 45. Burg JL, Grover CM, Pouletty P, Boothroyd JC. Direct and sensitive detection of a 713 pathogenic protozoan, Toxoplasma gondii, by polymerase chain reaction. J Clin Microbiol. 714 1989;27(8):1787-92. 

detection of Toxoplasma gondii. J Clin Microbiol. 2000;38(11):4121-5.

47. Butcher BA, Fox BA, Rommereim LM, Kim SG, Maurer KJ, Yarovinsky F, et al. Toxoplasma gondii rhoptry kinase ROP16 activates STAT3 and STAT6 resulting in cytokine inhibition and arginase-1-dependent growth control. PLoS Pathog. 2011;7(9):e1002236.

48. Baruch K, Deczkowska A, David E, Castellano JM, Miller O, Kertser A, et al. Aging. Aging-induced type I interferon response at the choroid plexus negatively affects brain function. Science. 2014;346(6205):89-93.

723

724

725

726

727

728

729

730

49. Baruch K, Ron-Harel N, Gal H, Deczkowska A, Shifrut E, Ndifon W, et al. CNSspecific immunity at the choroid plexus shifts toward destructive Th2 inflammation in brain aging. Proc Natl Acad Sci U S A. 2013;110(6):2264-9.

50. Kunis G, Baruch K, Rosenzweig N, Kertser A, Miller O, Berkutzki T, et al. IFNgamma-dependent activation of the brain's choroid plexus for CNS immune surveillance and repair. Brain. 2013;136(Pt 11):3427-40.

51. Helms HC, Abbott NJ, Burek M, Cecchelli R, Couraud PO, Deli MA, et al. In vitro models of the blood-brain barrier: An overview of commonly used brain endothelial cell culture models and guidelines for their use. J Cereb Blood Flow Metab. 2016;36(5):862-90.

52. Szmydynger-Chodobska J, Strazielle N, Gandy JR, Keefe TH, Zink BJ, Ghersi-Egea JF, et al. Posttraumatic invasion of monocytes across the blood-cerebrospinal fluid barrier. $\mathrm{J}$ Cereb Blood Flow Metab. 2012;32(1):93-104.

53. Solar P, Zamani A, Kubickova L, Dubovy P, Joukal M. Choroid plexus and the bloodcerebrospinal fluid barrier in disease. Fluids Barriers CNS. 2020;17(1):35.

54. Shechter R, Miller O, Yovel G, Rosenzweig N, London A, Ruckh J, et al. Recruitment of beneficial M2 macrophages to injured spinal cord is orchestrated by remote brain choroid plexus. Immunity. 2013;38(3):555-69.

55. Schwartz M, Baruch K. The resolution of neuroinflammation in neurodegeneration: leukocyte recruitment via the choroid plexus. EMBO J. 2014;33(1):7-22.

56. Kunis G, Baruch K, Miller O, Schwartz M. Immunization with a Myelin-Derived Antigen Activates the Brain's Choroid Plexus for Recruitment of Immunoregulatory Cells to the CNS and Attenuates Disease Progression in a Mouse Model of ALS. J Neurosci. 2015;35(16):6381-93.

57. Dando SJ, Mackay-Sim A, Norton R, Currie BJ, St John JA, Ekberg JA, et al. Pathogens penetrating the central nervous system: infection pathways and the cellular and molecular mechanisms of invasion. Clin Microbiol Rev. 2014;27(4):691-726.

58. Mohle L, Parlog A, Pahnke J, Dunay IR. Spinal cord pathology in chronic experimental Toxoplasma gondii infection. Eur J Microbiol Immunol (Bp). 2014;4(1):65-75.

59. Drewry LL, Jones NG, Wang Q, Onken MD, Miller MJ, Sibley LD. The secreted kinase ROP17 promotes Toxoplasma gondii dissemination by hijacking monocyte tissue migration. Nat Microbiol. 2019;4(11):1951-63. 
60. Serot JM, Zmudka J, Jouanny P. A possible role for CSF turnover and choroid plexus in the pathogenesis of late onset Alzheimer's disease. J Alzheimers Dis. 2012;30(1):17-26.

61. Meeker RB, Williams K, Killebrew DA, Hudson LC. Cell trafficking through the choroid plexus. Cell Adh Migr. 2012;6(5):390-6.

62. Ochiai E, Sa Q, Brogli M, Kudo T, Wang X, Dubey JP, et al. CXCL9 is important for recruiting immune $\mathrm{T}$ cells into the brain and inducing an accumulation of the $\mathrm{T}$ cells to the areas of tachyzoite proliferation to prevent reactivation of chronic cerebral infection with Toxoplasma gondii. Am J Pathol. 2015;185(2):314-24.

63. Meoli L, Gunzel D. Channel functions of claudins in the organization of biological systems. Biochim Biophys Acta Biomembr. 2020;1862(9):183344.

64. Kratzer I, Vasiljevic A, Rey C, Fevre-Montange M, Saunders N, Strazielle N, et al. Complexity and developmental changes in the expression pattern of claudins at the bloodCSF barrier. Histochem Cell Biol. 2012;138(6):861-79.

65. Bhat AA, Syed N, Therachiyil L, Nisar S, Hashem S, Macha MA, et al. Claudin-1, A Double-Edged Sword in Cancer. Int J Mol Sci. 2020;21(2).

66. Zhou B, Flodby P, Luo J, Castillo DR, Liu Y, Yu FX, et al. Claudin-18-mediated YAP activity regulates lung stem and progenitor cell homeostasis and tumorigenesis. J Clin Invest. 2018;128(3):970-84.

67. Saeedi BJ, Kao DJ, Kitzenberg DA, Dobrinskikh E, Schwisow KD, Masterson JC, et al. HIF-dependent regulation of claudin-1 is central to intestinal epithelial tight junction integrity. Mol Biol Cell. 2015;26(12):2252-62.

68. Kam KR, Walsh LA, Bock SM, Ollerenshaw JD, Ross RF, Desai TA. The effect of nanotopography on modulating protein adsorption and the fibrotic response. Tissue Eng Part A. 2014;20(1-2):130-8.

69. Samak G, Gangwar R, Crosby LM, Desai LP, Wilhelm K, Waters CM, et al. Cyclic stretch disrupts apical junctional complexes in Caco-2 cell monolayers by a JNK-2-, c-Src-, and MLCK-dependent mechanism. Am J Physiol Gastrointest Liver Physiol. 2014;306(11):G947-58.

70. Shrestha B, Paul D, Pachter JS. Alterations in tight junction protein and IgG permeability accompany leukocyte extravasation across the choroid plexus during neuroinflammation. J Neuropathol Exp Neurol. 2014;73(11):1047-61. Kinetic profile of the transcriptome changes induced in the choroid plexus by peripheral inflammation. J Cereb Blood Flow Metab. 2009;29(5):921-32. how African trypanosomes invade the CNS. Trends Parasitol. 2014;30(10):470-7. 

74. Leppert D, Leib SL, Grygar C, Miller KM, Schaad UB, Hollander GA. Matrix metalloproteinase (MMP)-8 and MMP-9 in cerebrospinal fluid during bacterial meningitis: association with blood-brain barrier damage and neurological sequelae. Clin Infect Dis. 2000;31(1):80-4.

75. Vandenbroucke RE, Dejonckheere E, Van Hauwermeiren F, Lodens S, De Rycke R, Van Wonterghem E, et al. Matrix metalloproteinase 13 modulates intestinal epithelial barrier integrity in inflammatory diseases by activating TNF. EMBO Mol Med. 2013;5(7):1000-16.

76. French T, Dusedau HP, Steffen J, Biswas A, Ahmed N, Hartmann S, et al. Neuronal impairment following chronic Toxoplasma gondii infection is aggravated by intestinal nematode challenge in an IFN-gamma-dependent manner. J Neuroinflammation. 2019;16(1):159.

\section{Figure Legends}

Fig1. Detection of $\boldsymbol{T}$. gondii in the CP. Mice were infected i.p. with 2 cysts of $T$. gondii type II ME49 and samples were collected at 5dpi. From brains, CPs were isolated under stereomicroscope, and the remaining brain tissue was processed for isolation of BMVs. (A) Representative image of freshly isolated CP and a phase-contrast image of isolated BMV. (B) Parasite burden $q \mathrm{PCR}$ analysis of isolated CPs (blue) and BMVs (red) from the correlated animals, which were infected at the same time, and analysed at 3,5 and 7dpi. The analysis was performed based on the presence of $B 1$ gene of $T$. gondii $(\operatorname{Tg} B 1)$ normalized to the murine gene $A s l$. Data were normalized to the mean values of 3dpi, and bar charts show individual values of a representative experiment, and mean + SEM. $n=5$ per group. Statistical analysis was performed by multiple $t$-test, with Holm-Sidak correction for multiple comparisons. ${ }^{* *} \mathrm{p}<0.01$. (C) CP whole mount staining to identify immune cells (CD45, blue), T. gondii (SAG1, red) and epithelial cells (E-cadherin, green). Scale bars $=50 \mu \mathrm{m}$ (D) BMVs immune-staining to identify pericytes (PDGFR $\beta$, blue), T. gondii (SAG1, red), and the tight junction ZO-1 (green). Yellow arrows indicate the co-localization of parasites with CD45+ immune cells, and yellow asterisks T. gondii signal alone. Scale bars $=50 \mu \mathrm{m}$.

Fig2. T. gondii infection of endothelial and immune cells in the CP. (A) Animals were infected i.p. with 1x10e5 T. gondii type II PTG-GFP tachyzoites. The brains were isolated at 
823 7dpi, and coronal sections were immune-stained with anti-GFP (green), anti-CD31 (magenta) 824 and DAPI (cyan). First row indicate the location of the CP (magenta rectangle) in the lateral 825 ventricle (VL) according to the mouse brain atlas. Second row magnify the CP area above. 826 Yellow arrowheads indicate the detection of parasites co-localized with endothelial cells 827 (CD31). Scale bars $=50 \mu \mathrm{m}(\mathbf{B})$ Isolated CPs from animals infected i.p. with 1x10e5 PTG-GFP 828 tachyzoites were immune-stained as whole-tissue mount with anti-CD45 (blue), anti-SAG1 829 (red), and anti-CD31 (green). Animals were infected at the same time, but tissues were analysed at 3, 5 and 7dpi. Yellow arrows indicate CD45/SAG1 co-localization, showing immune cells carrying parasites through the BCSFB. Arrowheads indicate co-localization of CD31/SAG1,

832 and asterisks represent SAG1 signal alone. GFP signal plus SAG1 staining were detected in the 833 same channel and coloured in red for better visualization. Scale bars $=50 \mu \mathrm{m}$. CTX $=$ cortex; $834 \mathrm{Cc}=$ corpus callosum; $\mathrm{CdP}=$ caudoputamen; $\mathrm{LS}=$ lateral septal; $\mathrm{VL}=$ lateral ventricle.

Fig3. Initial immune response in the $\mathrm{CP}$ and $\mathrm{BBB}$ upon $T$. gondii infection. Single live cells from (A-D) isolated CP and (E-H) brain were analysed by flow cytometry, and the expression of MHCII was evaluated on endothelial cells (CD31+CD45-) from both tissues. Dot plots (A,

838 E) represent the gating strategy based on FMO controls. Histograms (B, F) provide a visual representation of the temporal MHCII expression levels by endothelial cells over $T$. gondii early 840 phase of infection. Bar charts $(\mathbf{C}, \mathbf{G})$ show the frequency in $\%$ of cells derived from parent population (CD31+CD45-). Bar charts $(\mathbf{D}, \mathbf{H})$ show MFI values of MHCII expression on the 842 endothelial cells. RT-PCR of total RNA isolated from CP and BMV was performed for (I)

843 cytokine expression, (J) IFN $\gamma$-regulated host-defense factors, and (K) chemokines. Data from $844 \mathrm{C}, \mathrm{D}, \mathrm{G}, \mathrm{H}$ show individual values and mean $\pm \mathrm{SEM}, \mathrm{n}=5,{ }^{*} p<0.05,{ }^{* *} p<0.01,{ }^{* * *} p<$ $0.001, * * * * p<0.0001$ (one-way ANOVA, with Tukey's correction). Data from I, J, K was normalized to naïve (day 0) BMV mean, and individual values as mean \pm SEM are shown, $\mathrm{n}=$ 2-4, \# $p<0.05, \# \# p<0.01$, \#\#\# $p<0.001$, \#\#\#\# $p<0.0001$, (one-way ANOVA, with Dunnett's

848 correction). \# indicate significant difference between CP and BMV from the same time-point, 
849 and * indicate significant difference between day 0 and the time-point being analysed from the 850 same tissue.

Fig4. Dysregulated tight junctions in the CP affect BCSFB function during early infection.

852 (A) Tight junctions and (B) MMP-8, and MMP-13 expression analysis (RT-PCR) of total RNA 853 isolated from $\mathrm{CP}$ at 3, 5 and 7 dpi. Data show individual values and mean $\pm \mathrm{SEM}, \mathrm{n}_{-}=2-5,{ }^{*} p<$ $8540.05, * * p<0.01,{ }^{* * *} p<0.001, * * * * p<0.0001$ (one-way ANOVA, with Dunnett's correction).

855 (C) Immunofluorescence of coronal brain sections stained for identification of Claudin-2 856 (green) and ZO-1 (red) tight junctions from naïve and 7dpi mice. White squares identify the 857 regions of interest shown in higher magnification in the white upper squares. White arrows 858 indicate different areas of Claudin-2 disturbance. Scale bars $=50 \mu \mathrm{m}$ (D) Functional FITC859 dextran permeability assay comparing the leakage of the BCSFB (blue) versus BBB (red) 860 throughout the course of infection. Data show mean \pm SEM $(n=5)$ for each time point analysed. 861 $* * * * p<0.0001, \# p<0.05, \# \# \# \#<<0.0001$. \# indicate significant difference between BCSFB and BBB from the same time-point (multiple t-test), and * indicate significant differences 863 between day 0 and the time-point being analysed from the same barrier type (one-way ANOVA, 864 with Dunnett's correction).

865 Fig5. Epithelial CP cells response against $T$. gondii in vitro. (A) Representative image of 866 cultured choroid plexus epithelial cells stained for the tight junction ZO-1, and myeloid cell 867 marker IBA-1. (B) Flow cytometric validation of cultured epithelial cells, identified as E868 cadherin $^{+} \mathrm{CD} 45^{-} \mathrm{CD} 31^{-}$. (C-D) Primary cultures of CP epithelial cells were infected for $6 \mathrm{~h}$ with 869 reporter T. gondii type II PRU-GFP tachyzoites at MOI=5, and controls remained non-infected. 870 Cultivated cells were immune-stained for detection of (C) ZO-1 (red) and (D) Claudin-2 (red). 871 DAPI stained the nucleus (blue). GFP-reporter T. gondii (green). Scale bars $=50 \mu \mathrm{m}$. (E-G) 872 Naïve and infected cultures were analysed for gene expression of $(\mathbf{E})$ cytokines and Ifngr 2, (F) 873 tight junctions, and (G) MMPs. Bar charts show individual mean values of triplicates from a 
874 representative experiment, and the mean $+\operatorname{SEM}(n=3)$ for each time point analysed. Data was 875 normalized by naïve means, besides $M m p 13$ which was not detectable on naïve samples ${ }^{*} p<$ $8760.05, * * p<0.01$ (student $t$-test).

\section{Additional files}

\begin{tabular}{|l|l|l|l|}
\hline File name & File format & Title of data & Description of data \\
\hline Additional file 1 & .docx & $\begin{array}{l}\text { Oligonucleotide primers used for } \\
\text { qPCR and RT-qPCR }\end{array}$ & - \\
\hline Additional file 2 & .tiff & $\begin{array}{l}\text { Detection of } T . \text { gondii on BMV, } \\
\text { brain with removed CP and Spinal } \\
\text { cord }\end{array}$ & legend below \\
\hline Additional file 3 & .tiff & $\begin{array}{l}\text { Detection of T. gondii in the CP } \\
\text { and brain }\end{array}$ & legend below \\
\hline Additional file 4 & .tiff & $\begin{array}{l}\text { MHCII expression by microglia } \\
\text { cells and CP epithelial cells }\end{array}$ & legend below \\
\hline Additional file 5 & .tiff & $\begin{array}{l}\text { Complementary gene expression } \\
\text { analysis of CP and BMVs }\end{array}$ & legend below \\
\hline
\end{tabular}

878

Additional file 2. Detection of $T$. gondii on BMV, brain with removed CP and Spinal cord.

Animals were infected i.p. with 2 cysts of T. gondii type II ME49. CPs were removed and the remaining brain tissue was processed for isolation of BMVs. (A) BMVs from 7dpi were stained

882 to identify pericytes (PDGFR $\beta)$, T. gondii (SAG1), and the tight junction ZO-1. White square 883 area is shown in higher magnification on the right image, and the yellow arrow points to the 884 disseminated signal for T. gondii. (B) Parasite burden in spleens of infected mice at 3, 5 and 7dpi. The analysis was performed based on the presence of $B 1$ gene of $T$. gondii $(\operatorname{TgB} 1)$

886 normalized to the murine gene Asl. Data were normalized to the mean values of $3 \mathrm{dpi}$, and bar 887 charts show individual values of a representative experiment, and mean $+\mathrm{SEM}, \mathrm{n}=4 .{ }^{*} \mathrm{p}<0.05$ 888 (multiple $t$-test, with Holm-Sidak correction). (C) Brains were isolated, CP removed, and remaining total brain homogenate was processed for parasite detection. (D) Spinal cords from 
890

891

892

893

894

895

896

897

898

899

900

901

902

903

904

905

906

907

908

909

910

911

912

913

infected mice were also used to quantify parasite burden. The analysis was performed based on expression of Sagl gene normalized to Hprt expression. Bar charts show individual values of a representative experiment, and mean $+\mathrm{SEM}, \mathrm{n}=4$ (multiple $t$-test, with Holm-Sidak correction).

Additional file 3. Detection of $T$. gondii in the $\mathrm{CP}$ and brain. Animals were infected i.p. with 2 cysts of $T$. gondii type II ME49. The brains were isolated, and coronal sections or CP whole mount were immune-stained with anti-SAG1 (light blue), anti-CD31 (red), anti-Ecadherin (green) and DAPI (dark blue). (A) Confocal image of parasite detection on endothelial cells at 7dpi. (B) Magnified region of interested previously identified by white square. (C) Parasites identified in the brain cortex, and white square region is magnified in (D). (E) Parasites on CP and adjacent brain ventricular areas at $7 \mathrm{dpi}$. (F) CP whole tissue mount from animals infected i.p. with 1x10e5 T. gondii type II PRU-tdTomato tachyzoites, showing detection of parasites inside immune and endothelial cells at 3dpi.

Additional file 4. MHCII expression by microglia cells and CP epithelial cells. Single cells suspensions were discriminated based on FSC-SSC parameters, singlets, and viable cells (Zombie NIR negative). (A) Brain cells were gated and identified as microglia $\left(\mathrm{CD} 11 \mathrm{~b}^{+} \mathrm{CD} 45^{\text {int }}\right), \mathrm{CD} 45+$ immune cells, and double negative $(\mathrm{DN})$ cells. (B) Choroid plexus cells were first divided in two main populations based on FSC-SSC. Bigger, viable cells were first defined as CD45-CD31- then positive for the CP epithelial cell marker TTR (transthyretin). Smaller, viable cells were gated as $\mathrm{CD} 45^{+} \mathrm{CD} 31^{-}$immune cells, and $\mathrm{CD} 45^{-} \mathrm{CD} 31^{+}$endothelial cells. (C) Representative contour plots showing microglia and (D) CP epithelial cells MHCII expression at $7 \mathrm{dpi}$. Bar charts represent the frequency in $\%$ of cells from parent population, and MFI values of MHCII expression levels. Data represent individual values and mean \pm SEM, $\mathrm{n}=5, * * p<0.01, * * * p<0.001, * * * * p<0.0001$ (one-way ANOVA, with Tukey's correction). 
914 Additional file 5. Complementary gene expression analysis of CP and BMVs. RT-PCR of

915 total RNA from isolated tissue, for (A) MHCII expression on CP, (B, C) expression of 916 interferon-beta-1 (Ifnb1) and macrophage colony-stimulating factor (Csfl), respectively, in 917 isolated CP and BMV. (D, E) Expression of fractalkine (Cx3cl1) and intercellular-adhesion918 molecule-1 (Icam1) on CP. Data show individual values and mean $+\mathrm{SEM}, \mathrm{n}=3-5, * p<0.05$, $919 * * p<0.01, * * * p<0.001, * * * * p<0.0001$ (A, D, E, one-way ANOVA with Tukey's correction; 920 B, C, one-way ANOVA with Dunnett's correction). 


\section{Figures}

A

Isolated CP

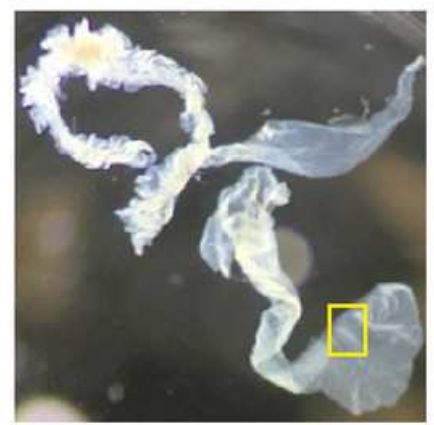

C

Isolated CP - 5dpi
B

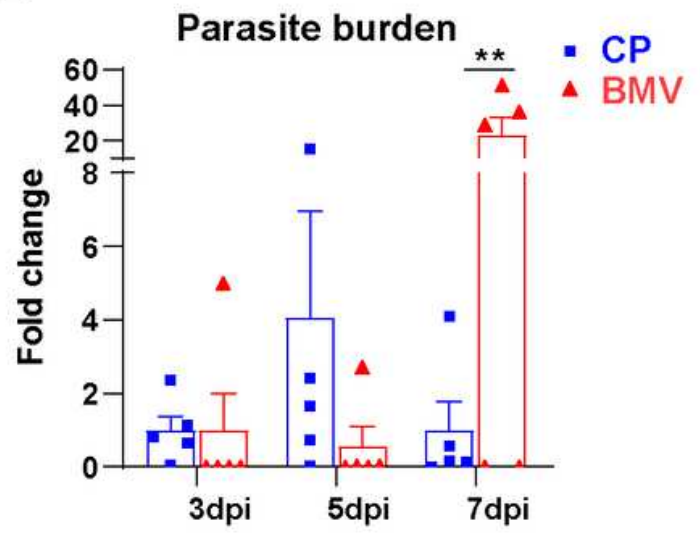

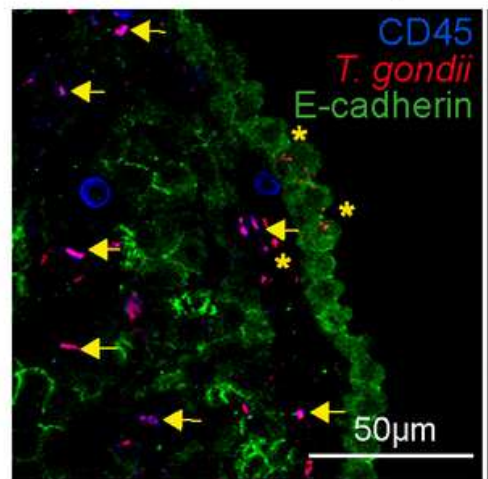
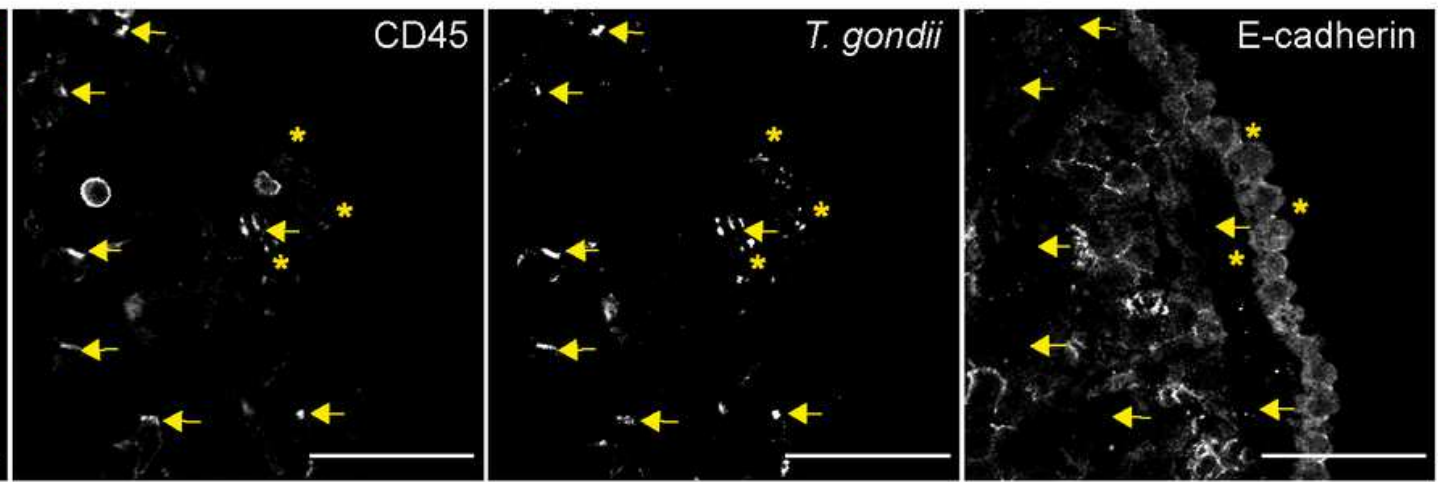

\section{Isolated BMV - 5dpi}
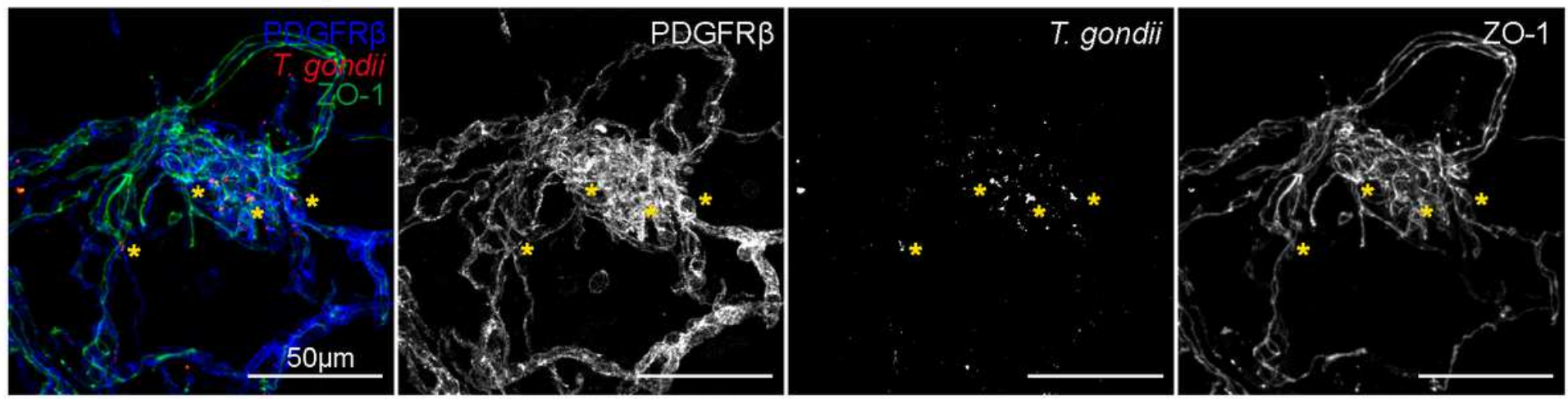

\section{Figure 1}

Detection of T. gondii in the CP. Mice were infected i.p. with 2 cysts of T. gondii type II ME49 and samples were collected at $5 \mathrm{dpi}$. From brains, CPs were isolated under stereomicroscope, and the remaining brain tissue was processed for isolation of BMVs. (A) Representative image of freshly isolated CP and a phasecontrast image of isolated BMV. (B) Parasite burden APCR analysis of isolated CPs (blue) and BMVs (red) from the correlated animals, which were infected at the same time, and analysed at 3, 5 and $7 \mathrm{dpi}$. The analysis was performed based on the presence of B1 gene of T. gondii (TgB1) normalized to the murine gene Asl. Data were normalized to the mean values of $3 \mathrm{dpi}$, and bar charts show individual values of a representative experiment, and mean + SEM. $n=5$ per group. Statistical analysis was performed by 
multiple t-test, with Holm-Sidak correction for multiple comparisons. ${ }^{* *} \mathrm{p}<0.01$. (C) CP whole mount staining to identify immune cells (CD45, blue), T. gondii (SAG1, red) and epithelial cells (E-cadherin, green). Scale bars $=50 \mu \mathrm{m}$ (D) BMVs immune-staining to identify pericytes (PDGFR $\beta$, blue), T. gondii (SAG1, red), and the tight junction ZO-1 (green). Yellow arrows indicate the co-localization of parasites with CD45+ immune cells, and yellow asterisks T. gondii signal alone. Scale bars $=50 \mu \mathrm{m}$.

A

\section{Brain coronal section - 7dpi}
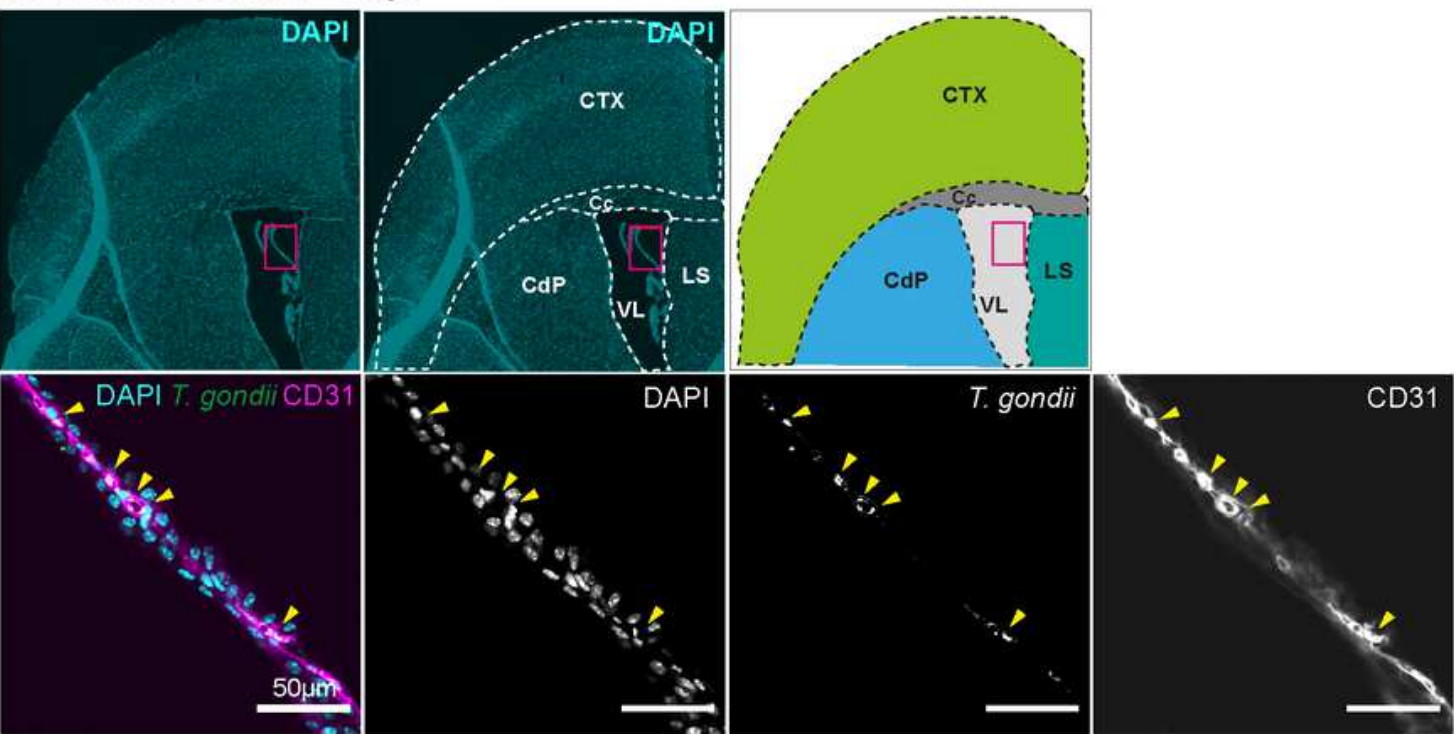

B

\section{Isolated CP}

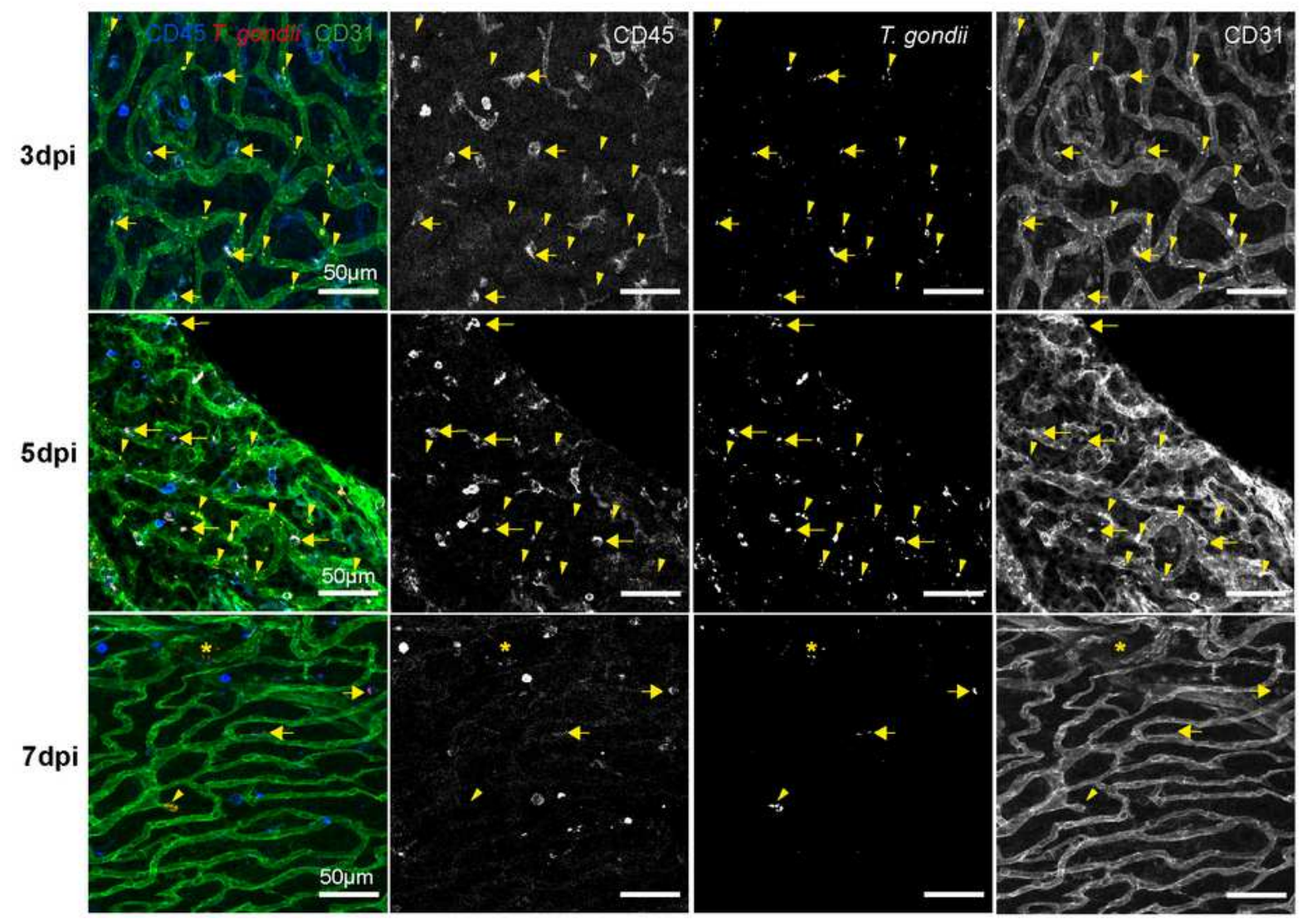

Figure 2 
T. gondii infection of endothelial and immune cells in the CP. (A) Animals were infected i.p. with 1x10e5 T. gondii type II PTG-GFP tachyzoites. The brains were isolated at 7dpi, and coronal sections were immunestained with anti-GFP (green), anti-CD31 (magenta) and DAPI (cyan). First row indicate the location of the $\mathrm{CP}$ (magenta rectangle) in the lateral ventricle (VL) according to the mouse brain atlas. Second row magnify the $\mathrm{CP}$ area above. Yellow arrowheads indicate the detection of parasites co-localized with endothelial cells (CD31). Scale bars $=50 \mu \mathrm{m}(\mathrm{B})$ Isolated CPs from animals infected i.p. with 1x10e5 PTGGFP tachyzoites were immune-stained as whole-tissue mount with anti-CD 45 (blue), anti-SAG1 (red), and anti-CD31 (green). Animals were infected at the same time, but tissues were analysed at 3, 5 and 7dpi. Yellow arrows indicate CD45/SAG1 co-localization, showing immune cells carrying parasites through the BCSFB. Arrowheads indicate co-localization of CD31/SAG1, and asterisks represent SAG1 signal alone. GFP signal plus SAG1 staining were detected in the same channel and coloured in red for better visualization. Scale bars $=50 \mu \mathrm{m}$. CTX=cortex; $\mathrm{Cc}=$ corpus callosum; $\mathrm{CdP}=$ caudoputamen; $\mathrm{LS}=$ lateral septal; VL= lateral ventricle.

A

Endothelial cells from $\mathrm{CP}$

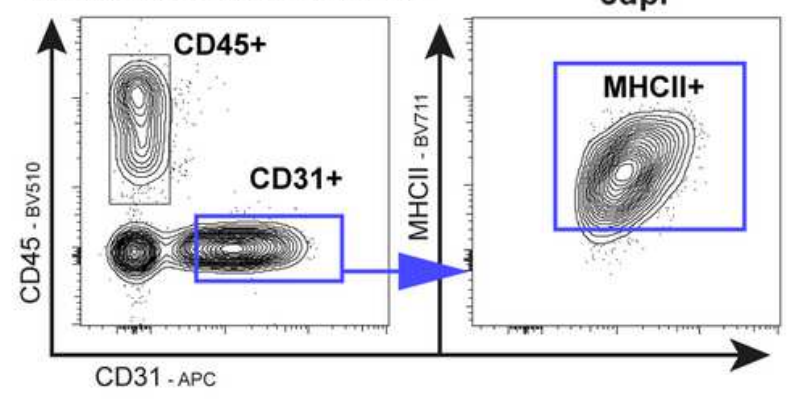

E

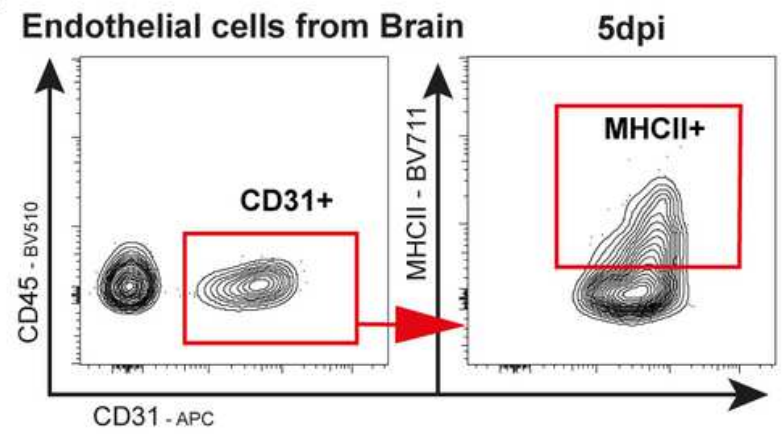

B

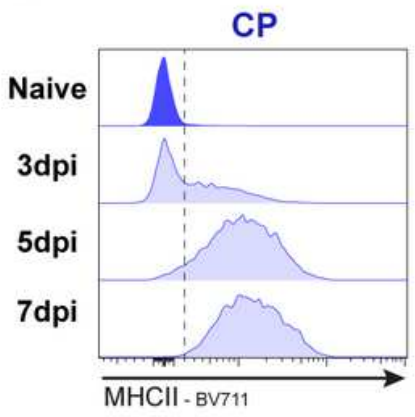

$\mathbf{F}$

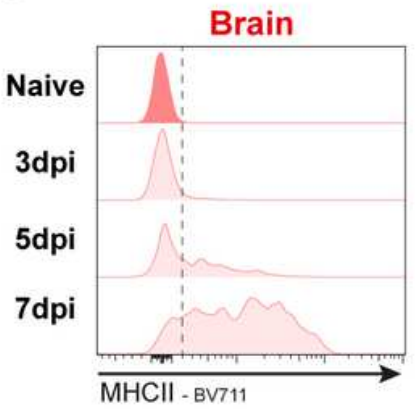

C

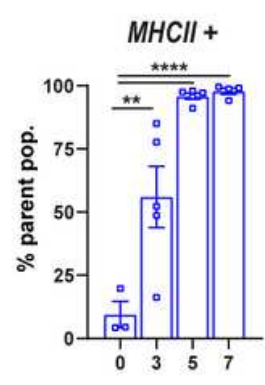

G

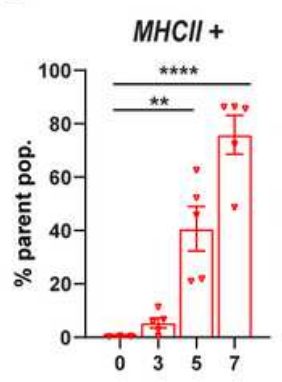

D

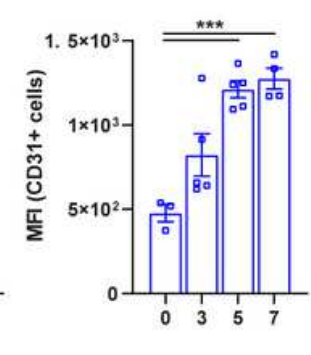

H

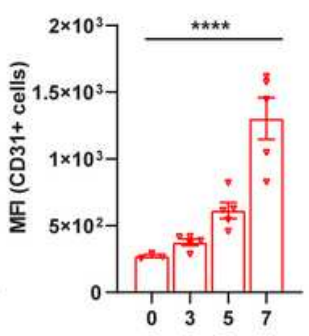

Gene expression in isolated CP and BMV

I
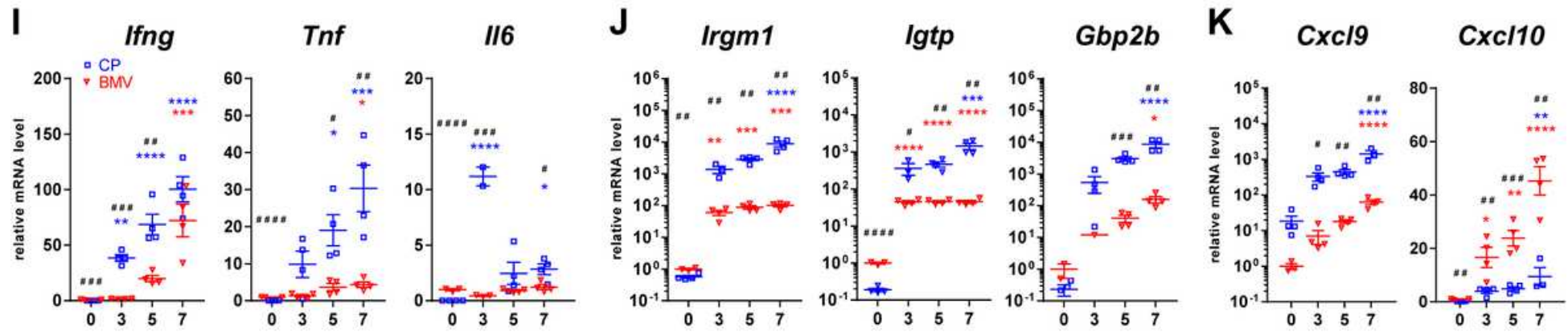

Figure 3 
Initial immune response in the CP and BBB upon T. gondii infection. Single live cells from (A-D) isolated $\mathrm{CP}$ and $(\mathrm{E}-\mathrm{H})$ brain were analysed by flow cytometry, and the expression of $\mathrm{MHCll}$ was evaluated on endothelial cells (CD31+CD45-) from both tissues. Dot plots $(A, E)$ represent the gating strategy based on FMO controls. Histograms (B, F) provide a visual representation of the temporal MHCll expression levels by endothelial cells over $T$. gondii early phase of infection. Bar charts $(C, G)$ show the frequency in $\%$ of cells derived from parent population (CD31+CD45-). Bar charts $(D, H)$ show MFI values of MHCII expression on the endothelial cells. RT-PCR of total RNA isolated from CP and BMV was performed for (I) cytokine expression, (J) IFN -regulated host-defense factors, and $(K)$ chemokines. Data from $C, D, G, H$ show individual values and mean $\pm S E M, n=5,{ }^{\star} p<0.05$, ${ }^{\star \star} p<0.01$, ${ }^{\star \star *} p<0.001$, ${ }^{\star \star \star *} p<0.0001$ (oneway ANOVA, with Tukey's correction). Data from I, J, K was normalized to naïve (day 0) BMV mean, and

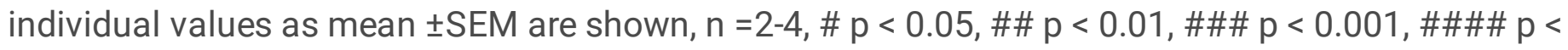
0.0001, (one-way ANOVA, with Dunnett's correction). \# indicate significant difference between CP and BMV from the same time-point, and * indicate significant difference between day 0 and the time-point being analysed from the same tissue.

\section{Gene expression in isolated CP}

A
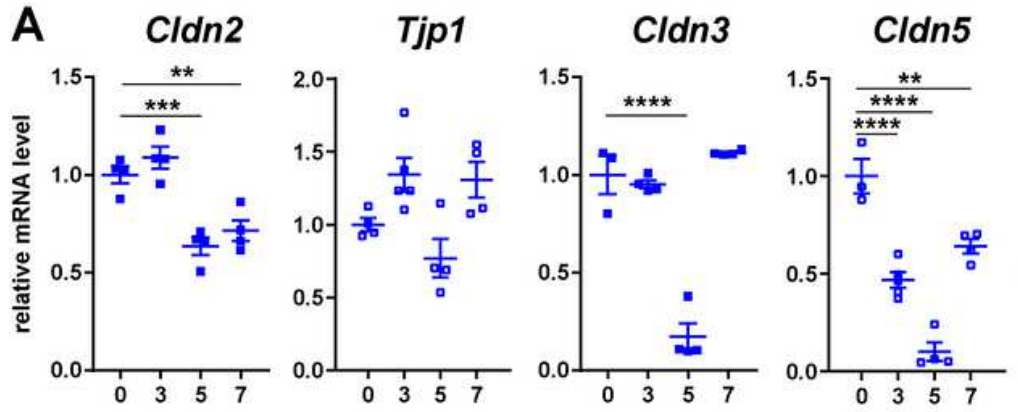

\section{Brain coronal section}

C
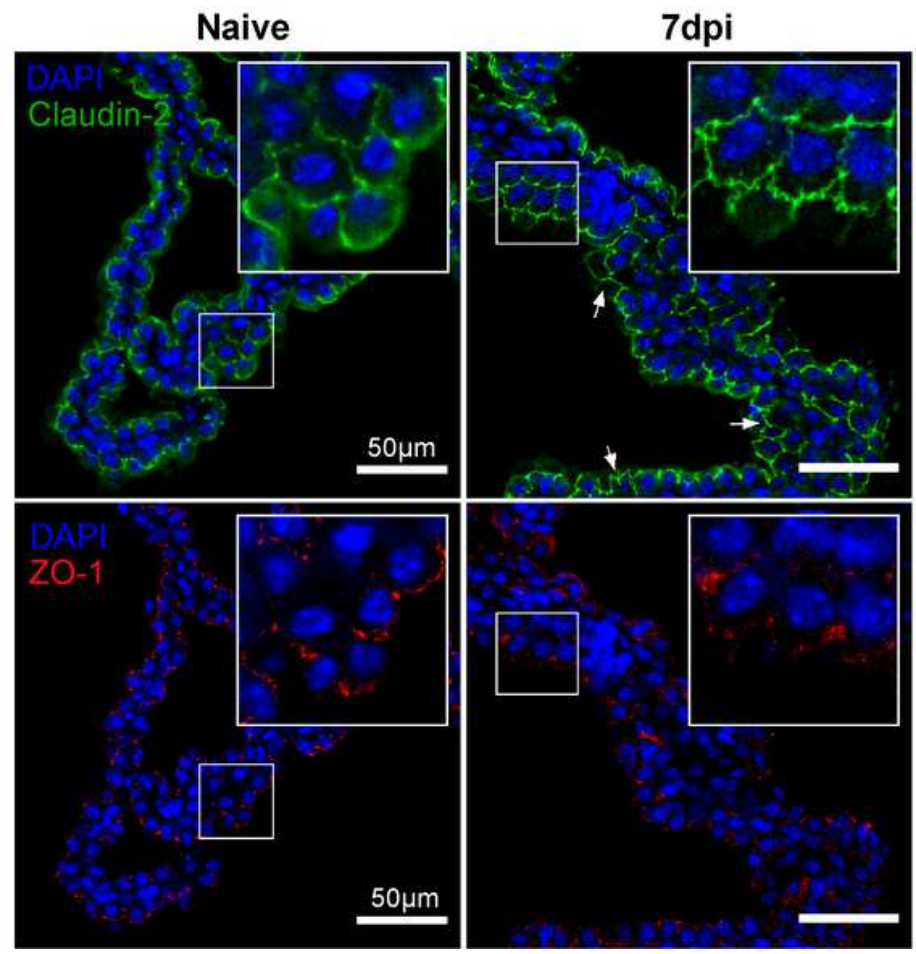
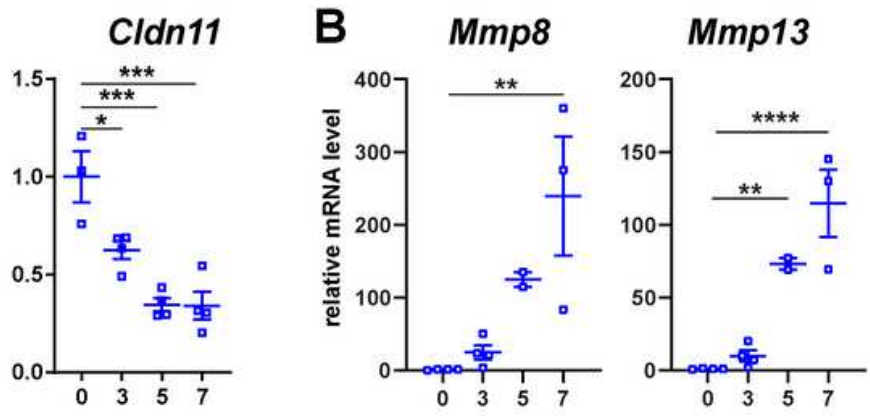

FITC-dextran Permeability assay

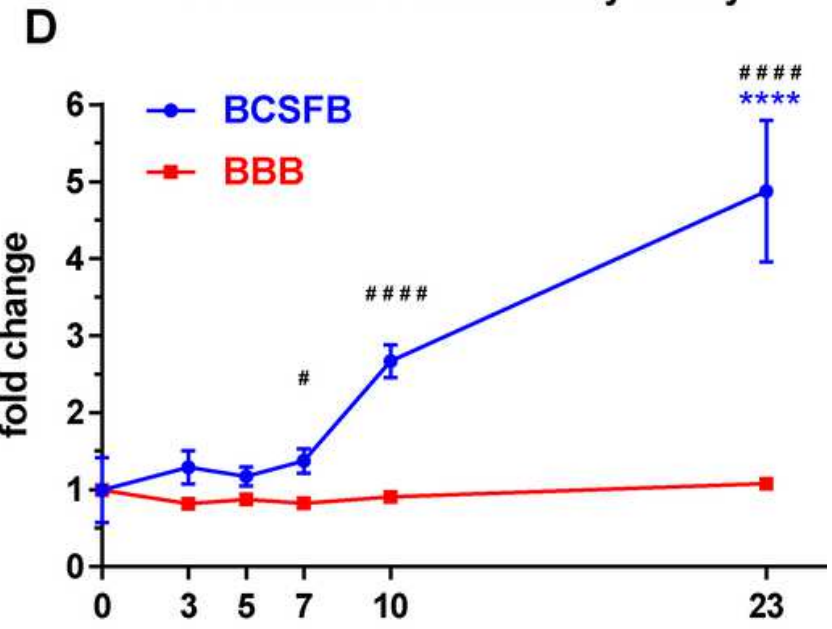


Dysregulated tight junctions in the CP affect BCSFB function during early infection. (A) Tight junctions and (B) MMP-8, and MMP-13 expression analysis (RT-PCR) of total RNA isolated from CP at 3, 5 and

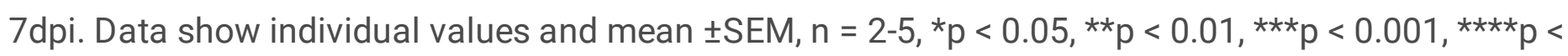
0.0001 (one-way ANOVA, with Dunnett's correction). (C) Immunofluorescence of coronal brain sections stained for identification of Claudin-2 (green) and ZO-1 (red) tight junctions from naïve and 7dpi mice. White squares identify the regions of interest shown in higher magnification in the white upper squares. White arrows indicate different areas of Claudin-2 disturbance. Scale bars $=50 \mu \mathrm{m}$ (D) Functional FITCdextran permeability assay comparing the leakage of the BCSFB (blue) versus BBB (red) throughout the course of infection. Data show mean \pm SEM $(n=5)$ for each time point analysed. ${ }^{\star \star \star *} p<0.0001, \# p<$ 0.05 , \#\#\#\# $<<0.0001$. \# indicate significant difference between BCSFB and BBB from the same timepoint (multiple t-test), and * indicate significant differences between day 0 and the time-point being analysed from the same barrier type (one-way ANOVA, with Dunnett's correction). 
Cells in the CP Epithelial culture

A

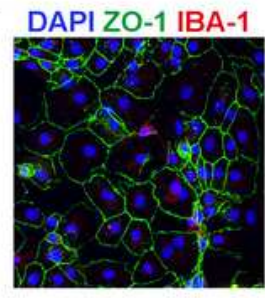

B

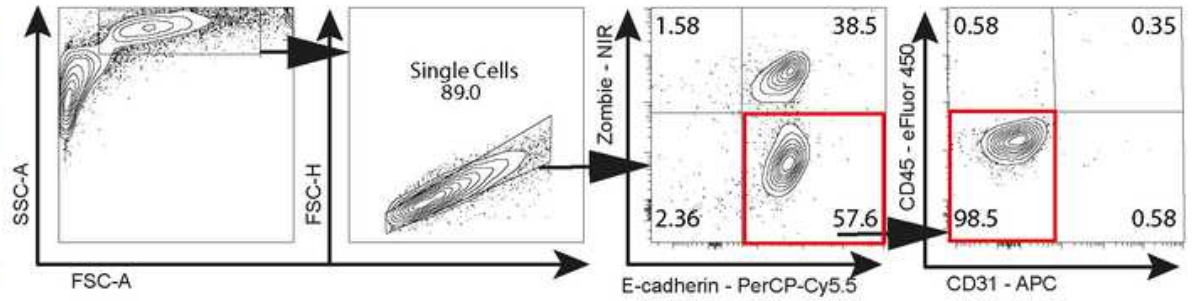

T. gondii infection of CP Epithelial culture in vitro

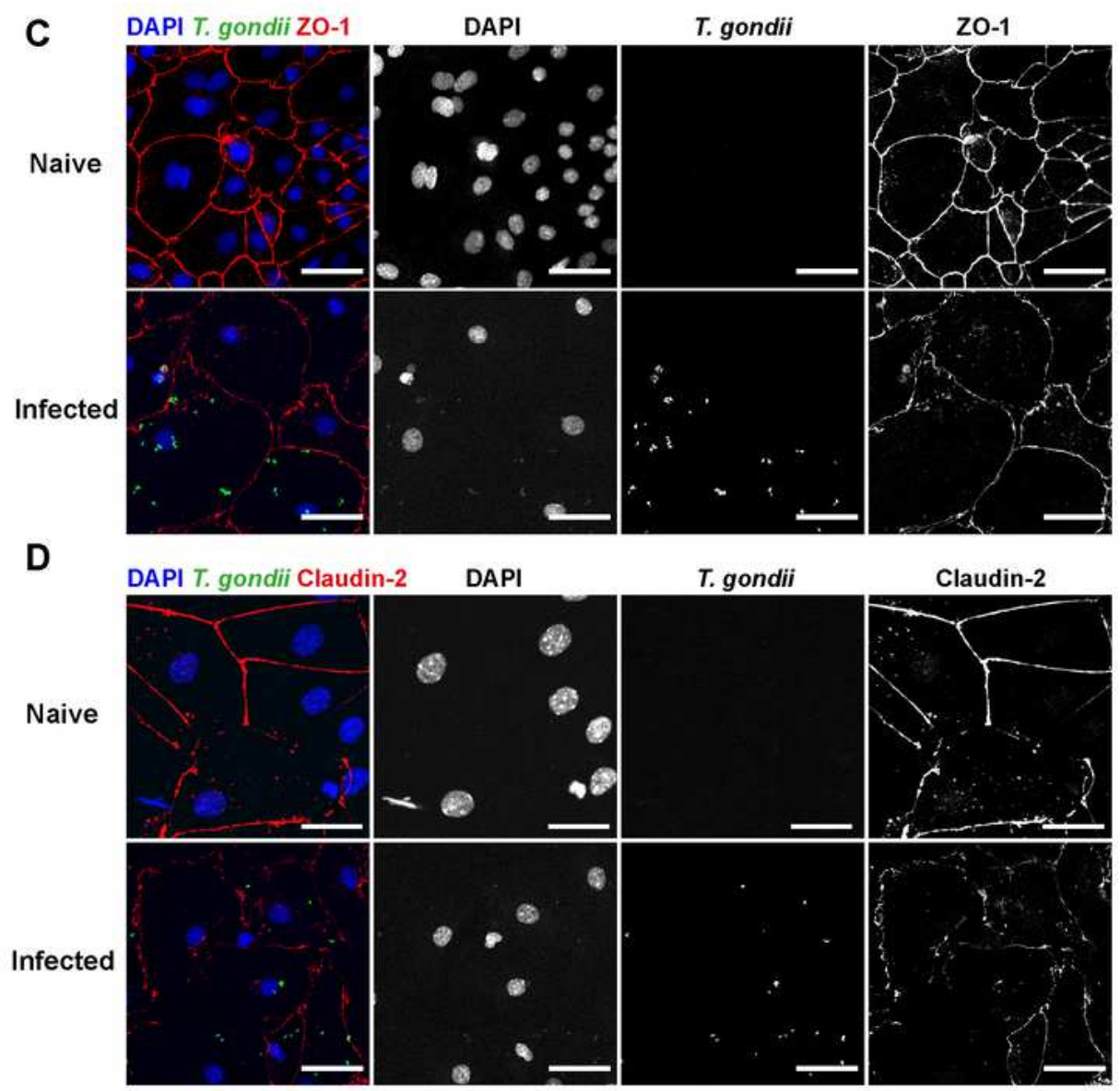

Gene expression in infected Epithelial cells in vitro

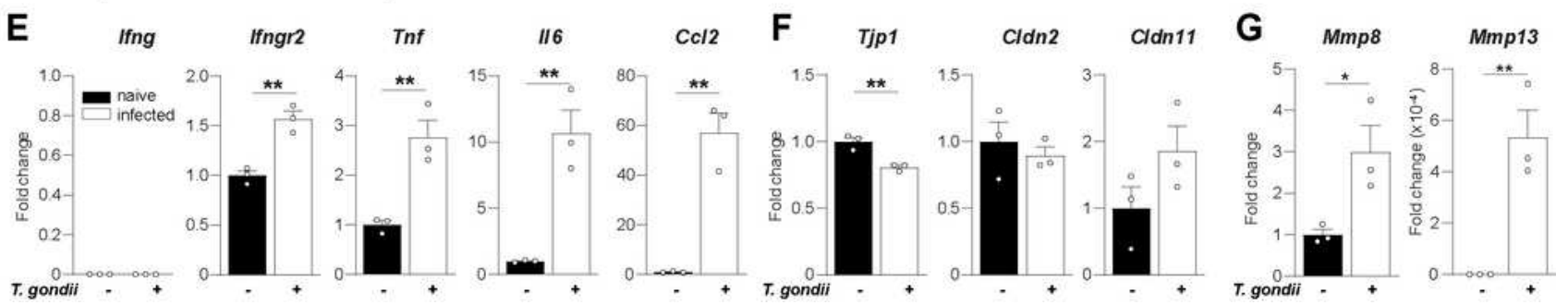

\section{Figure 5}

Epithelial CP cells response against T. gondii in vitro. (A) Representative image of cultured choroid plexus epithelial cells stained for the tight junction ZO-1, and myeloid cell marker IBA-1. (B) Flow cytometric validation of cultured epithelial cells, identified as E-cadherin+CD45-CD31-. (C-D) Primary cultures of CP epithelial cells were infected for $6 \mathrm{~h}$ with reporter T. gondii type II PRU-GFP tachyzoites at MOI=5, and controls remained non-infected. Cultivated cells were immune-stained for detection of (C) ZO-1 (red) and 
(D) Claudin-2 (red). DAPI stained the nucleus (blue). GFP-reporter T. gondii (green). Scale bars $=50 \mu \mathrm{m}$. (EG) Naïve and infected cultures were analysed for gene expression of (E) cytokines and Ifngr2, (F) tight junctions, and (G) MMPs. Bar charts show individual mean values of triplicates from a representative experiment, and the mean +SEM $(n=3)$ for each time point analysed. Data was normalized by naïve means, besides Mmp13 which was not detectable on naïve samples ${ }^{\star} p<0.05$, ${ }^{\star \star} p<0.01$ (student $t$-test).

\section{Supplementary Files}

This is a list of supplementary files associated with this preprint. Click to download.

- Additionalfile01tableofoligonucleotides.docx

- Additionalfile02.tif

- Additionalfile03.tif

- Additionalfile04.tif

- Additionalfile05.tif 